TRANSACTIONS OF THE

AMERICAN MATHEMATICAL SOCIETY

Volume 363, Number 6, June 2011, Pages 3309-3334

S 0002-9947(2011)05222-0

Article electronically published on January 11, 2011

\title{
WEAK EHRENFEUCHT-FRAÏSSÉ GAMES
}

\author{
TAPANI HYTTINEN AND VADIM KULIKOV
}

\begin{abstract}
In this paper we define a game which is played between two players I and II and two mathematical structures $\mathcal{A}$ and $\mathcal{B}$. The players choose elements from both structures in $\alpha$ moves, and at the end of the game player II wins if the chosen structures are isomorphic. Thus the difference between this and the ordinary Ehrenfeucht-Fraïssé game is that the isomorphism can be arbitrary, whereas in the ordinary EF-game it is determined by the moves of the players. We investigate determinacy of the weak EF-game for different $\alpha$ (the length of the game) and its relation to the ordinary EF-game.
\end{abstract}

\section{INTRODUCTION}

1.1. History and motivation. The following question arises very often in mathematics: Does a given description of a mathematical structure describe the structure up to isomorphism? Or equivalently: Is the structure satisfying given conditions unique? Also, if it is unique, can we further weaken the description or the conditions? Or if it is not unique, then how good is the description? Model theory and mathematical logic in general have a long history in studying these questions. In particular, classifying the ways of description which never lead to a unique solution, studying how much information these descriptions provide, studying various equivalence relations between structures which are weaker than (but as close as possible to) isomorphism, constructing strongly equivalent non-isomorphic models and giving methods to establish such weak equivalences between structures which under some conditions may lead to a unique description.

On the other hand, mathematicians often seek methods to distinguish between structures (invariants), which would be mathematically simple but which would still classify the structures of a certain class well enough. In many cases, for example, the isomorphism type is too hard an invariant, although it is the best possible for distinguishing structures. If one can show that a strong invariant does not distinguish between structures in a certain class of structures, then one knows that any invariant that would distinguish should be even more powerful. Winning strategies in various Ehrenfeucht-Fraïssé games provide such invariants.

One of the most celebrated problems solved that was in this area, and which was also one of the starting points for further investigation, was Whitehead's problem, which asks whether all Whitehead groups 1 are free abelian. Saharon Shelah proved

Received by the editors October 17, 2008 and, in revised form, October 11, 2009.

2010 Mathematics Subject Classification. Primary 03C55; Secondary 03C52.

The first author was partially supported by the Academy of Finland, grant 1106753

${ }^{1} \mathrm{~A}$ group $G$ is Whitehead if it is abelian and: For all abelian groups $B$ and surjective homomorphism $f: B \rightarrow G$ with $\operatorname{ker}(f) \cong \mathbb{Z}$ there exists a homomorphism $g: G \rightarrow B$ with $f \circ g=\operatorname{id}_{G}$.

(C)2011 American Mathematical Society Reverts to public domain 28 years from publication 
in 1974 that the answer is independent of ZFC. A similar question that has been studied is whether an almost free group is free. An almost free group is such a group that all its countable subgroups (or more generally, all subgroups of size $<\kappa$ for $\kappa$ an uncountable cardinal) are free, and similarly for free and almost free abelian groups. Many other properties of free and almost free groups are studied in this context; they also appear in the present article (Section 5).

In the 1950s A. Ehrenfeucht and R. Fraïssé introduced back-and-forth systems and what we call the Ehrenfeucht-Fraïssé games. They showed in particular that player $\boldsymbol{I}$ has a winning strategy in this game of length $n<\omega$ on structures $\mathcal{A}$ and $\mathcal{B}$ in a finite relational vocabulary if and only if the structures satisfy exactly the same first-order formulas of quantifier rank $n$. Carol Karp proved in 1965 that having a winning strategy (of player $\mathbf{I}$ ) in an EF-game of length $\omega$ is equivalent to $L_{\infty \omega}$-equivalence. These characterizations have already proved to be very useful. Instead of the property where the structures satisfy the same $L_{\infty}$-formulas, which is very subtle and difficult to handle, we have back-and-forth systems or winning strategies, which are (almost) always easier and more intuitive concepts.

In 1977, Kueker introduced countable approximations which are closely related (as we shall see in Section 4) to EF-games. Kueker studied how much information about a model can be obtained by looking at its countable submodels. It turns out that two structures have a closed unbounded set of isomorphic countable substructures if and only if they are $L_{\infty}$-equivalent, which by the above discussion is equivalent to having a winning strategy for player $\boldsymbol{\Pi}$ in the EF-game of length $\omega$.

There is a natural modification of EF-games (the weak EF-games), which in a special case gives an equivalence to Kueker's approach. This modified game however seems easier for player $\Pi$, i.e. provides a weaker equivalence. But in fact it is not the case (see Theorem 4.1).

The concept of weak EF-games is a natural modification of EF-games, also in a more technical way: In literature when one proves that player $\mathbf{I}$ wins the EF-game of some length, the given argument is often stronger than is actually needed - it in fact provides a winning strategy for him already in one of the weak EF games (it depends on the situation which one). This raises in particular the question as to whether the games are equivalent.

This article can be seen as an investigation of the idea of this new game, generalizing the concept of countable approximations to "uncountable approximations", giving new viewpoints on characterizations of equivalences, introducing new similarity relations between structures and finally constructing models with interesting properties with respect to the given similarities. For example, we give a method to construct structures on which the weak game of length $\kappa$ can be non-determined for certain $\kappa$, and this method also provides structures with non-reflecting winning strategies (see Section 6).

The authors wish to express their gratitude to Jouko Väänänen who suggested the topic of this paper.

1.2. The weak game and a sketch of the results. We introduce a similarity 2 relation on the class of first order $L$-structures for some vocabulary $L$. We define a two player game, the weak Ehrenfeucht-Fraïssé game, which defines this relation in the same manner as the ordinary Ehrenfeucht-Fraïssé game defines the

\footnotetext{
${ }^{2}$ We use the word similarity relation instead of equivalence relation, because the relations we consider are not necessarily transitive.
} 
EF-similarity relations 3 In the weak Ehrenfeucht-Fraïssé game of length $\alpha$ on structures $\mathcal{A}$ and $\mathcal{B}$, players $\mathbf{I}$ and $\boldsymbol{I}$ choose points from both structures. In the end player $\boldsymbol{I}$ wins if and only if the chosen substructures of size $\leqslant|\alpha|$ are isomorphic; notably the isomorphism can be arbitrary to contrast the ordinary EF-game. We denote the weak EF-game of length $\alpha$ on structures $\mathcal{A}$ and $\mathcal{B}$ by $\operatorname{EF}_{\alpha}^{*}(\mathcal{A}, \mathcal{B})$.

In the case of game length $\omega$, the question of whether $\mathrm{EF}_{\omega}^{*}$ is determined and whether it has any difference to the ordinary Ehrenfeucht-Fraïssé game was essentially solved - in a somewhat different context and formulation - in Kue. Our proof however does not rely on Kueker's, and we carried it out before we actually noticed the coincidence. It turns out that a player wins $\mathrm{EF}_{\omega}$ if and only if he or she wins $\mathrm{EF}_{\omega}^{*}$, and since $\mathrm{EF}_{\omega}$ is determined, $\mathrm{EF}_{\omega}^{*}$ is also determined.

Using this game we are able to generalize Kueker's equivalence relation to longer games. In fact, we define two weak games. The other one is denoted $\mathrm{EF}^{\circ} . \mathrm{EF}^{\circ}$ is weaker than $\mathrm{EF}$, and $\mathrm{EF}^{*}$ is weaker than $\mathrm{EF}^{\circ}$. We concentrated more on studying $\mathrm{EF}^{*}$, because it has clear model theoretic and set theoretic interpretations (see Theorem 2.14 and Section 5 . where a connection to the cub-game is drawn), it is easier to study and, most importantly, since the game $\mathrm{EF}^{\circ}$ falls in between the two other games, many results for $\mathrm{EF}^{*}$ imply results for $\mathrm{EF}^{\circ}$. When we say the weak EF-game, we mean $\mathrm{EF}^{*}$.

To sum up, we give the results listed below. If for any $X \in\{\mathbf{I}, \boldsymbol{\Pi}\}$ we have that $X$ wins the game $G$ if and only if $X$ wins $G^{\prime}$, then we say that these games $G$ and $G^{\prime}$ are equivalent, and if not, we say that they are different.

- (Theorem 3.2) If $\kappa^{<\lambda}=\kappa$, then if player $\mathbf{I}$ has a winning strategy in $\operatorname{EF}_{\lambda}(\mathcal{A}, \mathcal{B})$, then he also has it in $\operatorname{EF}_{\kappa}^{*}(\mathcal{A}, \mathcal{B})$.

- (Theorem 4.1) The games $\mathrm{EF}_{\omega}$ and $\mathrm{EF}_{\omega}^{*}$ are equivalent.

- (Examples 4.2 and 4.3) If $\omega<\alpha<\omega_{1}$, then $\mathrm{EF}_{\alpha}^{*}$ is properly weaker than $\mathrm{EF}_{\alpha}$.

- (Corollary 5.2) It was shown in MekSheVää that if the existence of a certain large cardinal is consistent, then it is consistent that $\mathrm{CH}$ holds and $\mathrm{EF}_{\omega_{1}}$ is determined on structures of size $\leqslant \aleph_{2}$. This implies (using Theorem 3.2) that it is consistent that all the games $\mathrm{EF}_{\omega_{1}}, \mathrm{EF}_{\omega_{1}}^{\circ}$ and $\mathrm{EF}_{\omega_{1}}^{*}$ are equivalent on structures of size $\leqslant \aleph_{2}$ and are all determined.

- (Theorems 5.8 and 5.9) Assuming $\square_{\omega_{1}}$ in MekSheVää], groups $\mathcal{F}$ and $\mathcal{G}$ of cardinality $\aleph_{2}$ were constructed so that $\operatorname{EF}_{\omega_{1}}(\mathcal{F}, \mathcal{G})$ is not determined. On these structures $\mathrm{EF}_{\omega_{1}}^{*}$ is determined and $\Pi$ wins. This is easy to generalize to $\square_{\kappa}$ and $\mathrm{EF}_{\kappa}, \mathrm{EF}_{\kappa}^{*}$.

- (Theorems 5.10, 5.11, 5.15 and 5.14) Using these structures $\mathcal{F}$ and $\mathcal{G}$ we can construct structures $\mathcal{F}^{\prime}, \mathcal{G}^{\prime}, M(\mathcal{F})$ and $M(\mathcal{G})$ (under GCH all are of cardinality $\left.\aleph_{2}\right)$ such that $\mathrm{EF}_{\omega_{1}}\left(\mathcal{F}^{\prime}, \mathcal{G}^{\prime}\right)$ is non-determined, but player $\Pi$ wins $\mathrm{EF}_{\omega_{1}}^{\circ}\left(\mathcal{F}^{\prime}, \mathcal{G}^{\prime}\right)$; the game $\mathrm{EF}_{\omega_{1}}^{\circ}(M(\mathcal{F}), M(\mathcal{G}))$ is non-determined, but $\boldsymbol{\Pi}$ wins $\mathrm{EF}_{\omega_{1}}^{*}(M(\mathcal{F}), M(\mathcal{G}))$.

- (Theorem 5.21) It is consistent with ZFC that there are structures $\mathcal{A}$ and $\mathcal{B}$ of cardinality $\aleph_{2}$ such that $\mathrm{EF}_{\omega_{1}}^{*}(\mathcal{A}, \mathcal{B})$ is not determined.

- (Theorem [5.22) In ZFC, there are structures $\mathcal{A}$ and $\mathcal{B}$ (of course larger than $\left.\aleph_{2}\right)$ such that $\mathrm{EF}_{\omega_{1}}^{*}(\mathcal{A}, \mathcal{B})$ is non-determined.

\footnotetext{
${ }^{3}$ The relations being "player $\mathbf{I}$ does not have a winning strategy in the EF game between $\mathcal{A}$ and $\mathcal{B}$ " and "player $\mathbf{I}$ has a winning strategy in the EF game between $\mathcal{A}$ and $\mathcal{B}$ ".
} 
- (Example 4.4 and Theorems 6.1, 6.2) In ZFC there are structures $\mathcal{A}$ and $\mathcal{B}$ such that player $\boldsymbol{\Pi}$ has a winning strategy in $\operatorname{EF}_{\beta}^{*}(\mathcal{A}, \mathcal{B})$ but not in $\operatorname{EF}_{\alpha}^{*}(\mathcal{A}, \mathcal{B})$, where $\alpha<\beta$ are ordinal numbers. It is consistent with ZFC that the above holds with $\alpha$ and $\beta$ both being cardinals.

\section{NotATION}

In this paper structures are ordinary structures of a first-order vocabulary $L$ unless stated otherwise. They are denoted by letters $\mathcal{A}, \mathcal{B}, \mathcal{C}$, and their domains, respectively, by $A, B, C$. Also, $\operatorname{dom}(\mathcal{A})$ is the domain of $\mathcal{A}$. If $f: X \rightarrow Y$ is a function, we denote $X=\operatorname{dom}(f)$, the domain of $f, f[A]$ or $f A$, the image of a set $A \subset X$, as well as $f^{-1} B=f^{-1}[B]$ as the inverse image of a set $B \subset Y$. Range is denoted as $\operatorname{ran}(f)=f[X]$.

We use standard notation from cardinal arithmetic, e.g. $\kappa^{<\lambda}=\bigcup_{\alpha<\lambda} \kappa^{\alpha}$ for $\kappa$ and $\lambda$ cardinals, and $A^{B}$ means the set of all functions with domain $B$ and range a subset of $A$. Sometimes, though, $\kappa^{\lambda}$ really means $\left|\kappa^{\lambda}\right|$, i.e. the cardinality of the set of all functions $\lambda \rightarrow \kappa$, but it should be clear from the context which one is being considered. There is no difference between $2^{\aleph_{0}}$ and $2^{\omega}$ except that when we write in the latter way, we want to emphasize that we are dealing with a well ordering rather than a cardinal number.

For a subset $A$ of an ordinal, $\operatorname{OTP}(A)$ (order type) denotes the ordinal which is order isomorphic to $A$. We follow MekSheVää, HytSheVää] and many others in the notation $S_{\lambda}^{\kappa}=\{\alpha<\kappa \mid \operatorname{cf}(\alpha)=\lambda\}$, and $\operatorname{cf}(\alpha)$ is the least ordinal which can be mapped into $\alpha$ cofinally, i.e. so that the image has no strict upper bound in $\alpha$.

\section{Definitions}

2.1. Definition. A game $G_{\gamma}(S)$ consists of a set $S$, game length $\gamma$ (an ordinal) and a winning set $W \subset(S \times S)^{\gamma}$. It is played between two players, I (he) and $\mathbf{I}$ (she). On the move $\beta<\gamma$ player $\mathbf{I}$ chooses $a_{\beta} \in S$ and then $\Pi$ chooses $b_{\beta} \in S$. Player $\boldsymbol{\Pi}$ wins if and only if $\left(a_{i}, b_{i}\right)_{i<\gamma} \in W$. Otherwise $\mathbf{I}$ wins.

2.2. Definition. Let $\mathcal{A}$ and $\mathcal{B}$ be structures and $\gamma$ an ordinal. The EhrenfeuchtFraïssé game of length $\gamma, \operatorname{EF}_{\gamma}(\mathcal{A}, \mathcal{B})$, is played as follows. On the move $\alpha, \alpha<\gamma$, player I chooses an element $a_{\alpha} \in A$ (or $b_{\alpha} \in B$ ). Then $\boldsymbol{I}$ answers by choosing an element $b_{\alpha} \in B$ (resp. $a_{\alpha} \in A$ ). Player $\Pi$ wins if the function $f$ which takes $a_{\alpha}$ to $b_{\alpha}$ for each $\alpha<\gamma$ is a partial isomorphism $\mathcal{A} \rightarrow \mathcal{B}$. Otherwise player I wins.

2.3. Definition. Let $\mathcal{A}, \mathcal{B}$ and $\gamma$ be as in Definition 2.2. The weak EhrenfeuchtFraïssé game of length $\gamma, \operatorname{EF}_{\gamma}^{*}(\mathcal{A}, \mathcal{B})$, is played as follows. At the move $\beta<\gamma$

Player I: chooses an element $a_{\beta} \in A \cup B$.

Player II: chooses an element $b_{\beta} \in A \cup B$.

Let $X=\left\{a_{\beta} \mid \beta<\gamma\right\} \cup\left\{b_{\beta} \mid \beta<\gamma\right\}$ be the set of all chosen elements. Player $\Pi$ wins if the substructures generated by $X \cap \mathcal{A}$ and $X \cap \mathcal{B}$ are isomorphic. Otherwise I wins.

Remark. Definition 2.3 makes sense only for relational vocabularies. If there are function symbols, then we should talk about the generated substructures $\langle X \cap \mathcal{A}\rangle$ and $\langle X \cap \mathcal{B}\rangle$ which might be more homogeneous and more likely isomorphic than the actual sets $X \cap \mathcal{A}$ and $X \cap \mathcal{B}$. That is why we use relational vocabularies throughout 
the paper except at one point (which may also demonstrate the difference), in Section 5 .

2.4. Definition. The game which is exactly as in Definition 2.3 , but where $\boldsymbol{\Pi}$ has to play from the different structure than $\mathbf{I}$ did on the same move, will be denoted $\mathrm{EF}_{\gamma}^{\circ}(\mathcal{A}, \mathcal{B})$.

By the weak Ehrenfeucht-Fraïssé game we will refer to the game $\mathrm{EF}^{*}$ defined in Definition 2.3. and by the weak EF-games we will refer to both $\mathrm{EF}^{*}$ and $\mathrm{EF}^{\circ}$.

2.5. Definition. A strategy for player $\mathbf{I}$ in some game $G_{\gamma}(S)$ is a function $\tau: S^{<\gamma} \rightarrow$ $S$. A strategy $\tau$ of player $\mathbf{I}$ is winning if player $\mathbf{I}$ always wins the game $G_{\gamma}(S)$ by playing the element $\tau\left(\left(b_{\alpha}\right)_{\alpha<\beta}\right)$ on the $\beta$ th move, where $b_{\alpha}$ are the elements that player $\boldsymbol{I}$ has chosen before the $\beta$ th move, for each $\beta<\gamma$. Analogously for player II.

Note that in the case of Ehrenfeucht-Fraïssé games on structures $\mathcal{A}$ and $\mathcal{B}$, a strategy is a function $\tau:(A \cup B)^{<\gamma} \rightarrow(A \cup B)$. A game is said to be determined if one of the players has a winning strategy; otherwise, it is said to be not determined or non-determined.

2.6. Definition. Assume that $\tau$ is a strategy for player $\mathbf{I}$ and $\sigma$ is a strategy for player $\boldsymbol{\Pi}$. Consider the game where $\mathbf{I}$ uses $\tau$ and $\boldsymbol{\Pi}$ uses $\sigma$. If $\boldsymbol{I}$ wins, we say that $\sigma$ beats $\tau$, and vice versa.

2.7. Lemma. A game $G$ is non-determined if and only if for every strategy $\tau$ of $\mathbf{I}$ there exists a strategy for $\boldsymbol{\Pi}$ that beats $\tau$, and for every strategy $\sigma$ of $\boldsymbol{\Pi}$ there exists a strategy for $\mathbf{I}$ that beats $\sigma$.

Proof. Straight from the definitions.

Let us introduce some notation that will be used throughout the paper:

$$
\begin{array}{rl}
X \uparrow G & \text { Player } X \text { has a winning strategy in the game } G, \\
\mathcal{A} \cong \mathcal{B} & \mathcal{A} \text { and } \mathcal{B} \text { are isomorphic, } \\
\mathcal{A} \sim_{\gamma} \mathcal{B} & \text { means the same as } \Pi \uparrow \mathrm{EF}_{\gamma}(\mathcal{A}, \mathcal{B}), \\
\mathcal{A} \sim_{\gamma}^{\circ} \mathcal{B} & \text { means the same as } \Pi \uparrow \operatorname{EF}_{\gamma}^{\circ}(\mathcal{A}, \mathcal{B}), \\
\mathcal{A} \sim_{\gamma}^{*} \mathcal{B} & \text { means the same as } \Pi \uparrow \operatorname{EF}_{\gamma}^{*}(\mathcal{A}, \mathcal{B}) .
\end{array}
$$

All of the relations, $\sim_{\gamma}, \sim_{\gamma}^{0}$ and $\sim_{\gamma}^{*}$ are equivalence relations on the class of $L$-structures.

It is clear that

$$
\Pi \uparrow \mathrm{EF}_{\gamma}(\mathcal{A}, \mathcal{B}) \Rightarrow \Pi \uparrow \mathrm{EF}_{\gamma}^{\circ}(\mathcal{A}, \mathcal{B}) \Rightarrow \Pi \uparrow \operatorname{EF}_{\gamma}^{*}(\mathcal{A}, \mathcal{B})
$$

and

$$
\mathbf{I} \uparrow \operatorname{EF}_{\gamma}(\mathcal{A}, \mathcal{B}) \Leftarrow \mathbf{I} \uparrow \operatorname{EF}_{\gamma}^{\circ}(\mathcal{A}, \mathcal{B}) \Leftarrow \mathbf{I} \uparrow \operatorname{EF}_{\gamma}^{*}(\mathcal{A}, \mathcal{B}) .
$$

The converses are those which are hard to prove or disprove.

An easy example shows that $\operatorname{EF}_{k}(\mathcal{A}, \mathcal{B})$ and $\operatorname{EF}_{k}^{*}(\mathcal{A}, \mathcal{B})$ are non-equivalent games for finite $k>1$.

2.8. Example. Let $A=\mathbb{N}$ and $B=\mathbb{Z}$ equipped with the usual ordering on both. Then $\mathbf{I}$ wins $\operatorname{EF}_{k}(\mathcal{A}, \mathcal{B})$ by first playing $0 \in \mathbb{N}$ and then $n-1 \in \mathbb{Z}$, where $n$ is the first move by $\boldsymbol{\Pi}$, so $\mathbf{I} \uparrow \mathrm{EF}_{k}(\mathcal{A}, \mathcal{B})$. On the other hand all finite linear orderings are 
isomorphic if and only if their cardinality is the same. Thus $\Pi \uparrow \operatorname{EF}_{k}^{\circ}(\mathcal{A}, \mathcal{B})$ and $\Pi \uparrow \operatorname{EF}_{k}^{*}(\mathcal{A}, \mathcal{B})$. In fact $\Pi \uparrow \operatorname{EF}_{k}^{*}(\mathcal{A}, \mathcal{B})$ holds for all $k<\omega$ and for linear orders $\mathcal{A}$ and $\mathcal{B}$.

Let us now turn our attention to infinite games. Let $\kappa$ be a cardinal. Consider the game $\operatorname{EF}_{\kappa}^{*}(\mathcal{A}, \mathcal{B})$. We will show that under the assumption $\kappa^{<\kappa}=\kappa$ player $\mathbf{I}$ has a winning strategy in $\operatorname{EF}_{\kappa}^{*}(\mathcal{A}, \mathcal{B})$ if and only if the set of isomorphic substructures of $\mathcal{A}$ and $\mathcal{B}$ of size $\kappa$ (see $(*)$ below) contains a $\kappa$-cub set, and player $\mathbf{I}$ has a winning strategy if and only if there is a $\kappa$-cub set of non-isomorphic such substructures. The used concepts will be defined first.

2.9. Definition. Let $(X,<)$ be a partial order. We say that a subset $C \subset X$ is a $\lambda$-cub if the following conditions are satisfied:

Closeness: Assume that $\left(c_{i}\right)_{i<\lambda}$ is an $<$-increasing chain of elements of $C$ and there exists an element $c \in X$ such that $\forall(i<\lambda)\left(c_{i}<c\right)$ and for all $c^{\prime} \in X$ if $c^{\prime}<c$, then $c^{\prime}<c_{i}$ for some $i<\lambda$. Then $c \in C$. The element $c$ is called the supremum of the chain $\left(c_{i}\right)_{i<\lambda}$.

Unboundedness: For each $c \in X$ there exists $c^{\prime} \in C$ such that $c<c^{\prime}$.

Notation. $[X]^{<\kappa^{+}}=\left\{Y \subset X|| Y \mid<\kappa^{+}\right\}$. This is not to be confused with the already used $(X)^{<\gamma}=\{f: \alpha \rightarrow X \mid \alpha<\gamma\}$. The set $[X]^{<\kappa^{+}}=\left\{Y \subset X|| Y \mid<\kappa^{+}\right\}$ equipped with the proper subset relation $Y<Y^{\prime} \Longleftrightarrow Y \subsetneq Y^{\prime}$ is a partially ordered set, and it is understood what is meant by a $\lambda$-cub subset of $[X]^{<\kappa^{+}}$. A set $C \subset[X]^{<\kappa^{+}}$is cub if it is $\lambda$-cub for all $\lambda<\kappa^{+}$.

Let $\mathcal{A}$ and $\mathcal{B}$ be two structures and let

$$
S=\{X \subset A \cup B|| X \mid \leqslant \kappa, X \cap \mathcal{A} \cong X \cap \mathcal{B}\} \subset[A \cup B]^{<\kappa^{+}} .
$$

Continuing this approach let us define:

2.10. Definition. Let $\mathcal{A}$ and $\mathcal{B}$ be some structures of the same vocabulary and $\lambda, \mu \leqslant \kappa$ non-zero cardinals, the length of the game $\kappa$ being infinite. Let us define the game $\operatorname{EF}_{\kappa}^{*}, \mu(\mathcal{A}, \mathcal{B})$ which is played between $\mathbf{I}$ and $\boldsymbol{\Pi}$ as follows. On the move $\alpha<\kappa$,

Player I: chooses $X_{\alpha} \subset A \cup B$ so that $\left|X_{\alpha}\right| \leqslant \lambda$, and then

Player II: chooses $Y_{\alpha} \subset A \cup B$ so that $\left|X_{\alpha}\right| \leqslant \mu$.

In the end $I$ wins if the substructures generated by $A \cap \bigcup_{\alpha<\kappa} X_{\alpha} \cup Y_{\alpha}$ and $B \cap$ $\bigcup_{\alpha<\kappa} X_{\alpha} \cup Y_{\alpha}$ are isomorphic. Otherwise $\mathbf{I}$ wins.

In Definition 2.3. $\mathrm{EF}_{\alpha}^{*}$ was defined for ordinals $\alpha$. We shall now see that when $\alpha=\kappa$ is an infinite cardinal, the defined games coincide.

2.11. Theorem. Let $\lambda, \mu$ and $\kappa$ be non-zero cardinals such that $\lambda, \mu \leqslant \kappa$ and $\kappa$ is infinite. Player $\mathbf{I}(\boldsymbol{\Pi})$ wins the game $\mathrm{EF}_{\kappa}^{*}, \mu(\mathcal{A}, \mathcal{B})$ if and only if he (she) wins the game $\mathrm{EF}_{\kappa}^{*}(\mathcal{A}, \mathcal{B})$.

Proof. Fix a bijective map $f: \kappa \times \kappa \rightarrow \kappa$ such that for each $\alpha$ we have $f(\alpha, \beta) \geqslant \alpha$.

Assume first that $\boldsymbol{\Pi}$ has a winning strategy in the game $\mathrm{EF}_{\kappa}^{\lambda, \mu}$. Then the strategy of $\Pi$ in $\operatorname{EF}_{\kappa}^{*}(\mathcal{A}, \mathcal{B})$ is as follows. She imagines that she is playing $\mathrm{EF}_{\kappa}^{*, \mu}$ against I. On each move she chooses $X_{\alpha} \subset A \cup B$ according to her strategy in the game $\mathrm{EF}_{\kappa}^{\lambda, \mu}$, and when he chooses an element $x_{\alpha} \in A \cup B$, she considers it as the set 
$\left\{x_{\alpha}\right\}$ being played by $\mathbf{I}$ in her imaginary game. Also, she enumerates all these sets $X_{\alpha}=\left\{x_{\alpha, \beta} \mid \beta<\kappa\right\}$ (enumeration need not be one-to-one), and on the $\gamma$ th move she plays $x_{f^{-1}(\gamma)}$ in the actual game. Thus she eventually picks the same set as she would in $\mathrm{EF}_{\kappa}^{*}, \mu$.

On the other hand, if $\Pi$ wins $\operatorname{EF}_{\kappa}^{*}(\mathcal{A}, \mathcal{B})$ the strategy for her in ${ }^{*} F_{\kappa}^{\lambda, \mu}$ is a reasoning somewhat converse to the previous: She imagines that they are playing $\mathrm{EF}_{\kappa}^{*}$. Every time he chooses a set $X_{\alpha} \in A \cup B$, she enumerates it, $X_{\alpha}=\left\{x_{\alpha, \beta} \mid\right.$ $\beta<\kappa\}$, and imagines that he played $x_{f^{-1}(\alpha)}$ in the game $\mathrm{EF}_{\kappa}^{*}$. In the actual game she plays $\left\{x_{\gamma}\right\}$, where $x_{\gamma}$ is according to the winning strategy in $\mathrm{EF}_{\kappa}^{*}$. Eventually the same sets are enumerated as if they were playing the imaginary game of $\boldsymbol{\Pi}$. So the resulting substructures are isomorphic, as she used a winning strategy.

The proofs for player $\mathbf{I}$ are completely analogous.

Remark. This shows that actually all games $\operatorname{EF}_{\kappa}^{*}, \mu(\mathcal{A}, \mathcal{B}), \lambda, \mu \leqslant \kappa$, are equivalent to the game $\mathrm{EF}_{\kappa}^{*}, \kappa(\mathcal{A}, \mathcal{B})$.

It is also not difficult to see that in $\operatorname{EF}_{\kappa}^{*}, \kappa(\mathcal{A}, \mathcal{B})$ we could require player $\Pi$ to choose on each move an $X \subset A \cup B$ such that $X \cap \mathcal{A} \cong X \cap \mathcal{B}$ and that it would not change the game (i.e. $\mathbb{I}$ wins exactly on the same structures as before, as well as I).

2.12. Lemma. Let $A$ be any set of size $>\kappa, \kappa$ a regular cardinal, and $\alpha$ an ordinal such that $\kappa^{<\alpha}=\left|\bigcup_{\beta<\alpha} \kappa^{\beta}\right|=\kappa$. Let $f: A^{<\alpha} \rightarrow A$ be any function. Then the set

$$
W=\{X \subset A \mid X \text { is closed under } f \text { and }|X| \leqslant \kappa\}
$$

is $\kappa$-cub in $[A]^{<\kappa^{+}}$.

Proof. We have to verify closeness $(i)$ and unboundedness (ii):

(i) If $X \in[A]^{\kappa^{+}}$, then by $\kappa^{<\alpha}=\kappa$ there exist $X^{\prime} \subset A$ such that $\left|X^{\prime}\right|=\kappa, X^{\prime}$ is closed under $\tau$ and $X \cup\{\tau(\varnothing)\} \subset X^{\prime}$. So $X<X^{\prime} \in W$.

(ii) Assume $\left(X_{\beta}\right)_{\beta<\kappa}$ is increasing and each $X_{\beta}$ is closed under $\tau$. To see that $\bigcup_{\beta<\kappa} X_{\beta}$ is also closed under $\tau$, let $k \in\left(\bigcup_{\beta<\kappa} X_{\beta}\right)^{<\alpha}$. Then $k \in\left(X_{\beta}\right)^{\gamma}$ for some $\beta<\kappa$ and $\gamma<\alpha \leqslant \kappa$, but $X_{\beta}$ is closed under $\tau$.

2.13. Lemma. Let $\tau$ (resp. $\sigma$ ) be a winning strategy for $\mathbf{I}$ (resp. II) in any of the three Ehrenfeucht-Fraïssé games of length $\kappa$, and suppose $X \subset A \cup B$ is closed under $\tau$ (resp. $\sigma$ ) and $|X|=\kappa$. Then $X \cap \mathcal{A} \neq X \cap \mathcal{B}$ (resp. $X \cap \mathcal{A} \cong X \cap \mathcal{B})$.

Proof. Assume first that $\tau$ is a winning strategy for $\mathbf{I}$.

We will show that if $X=Y \cup Z(Y \subset A, Z \subset B)$, then $Y$ and $Z$ cannot be isomorphic. Assume without loss of generality that $\tau$ is a winning strategy for $\mathbf{I}$ in $\mathrm{EF}_{\kappa}(\mathcal{A}, \mathcal{B})$. If there were an isomorphism $f: Y \cong Z$, then $\boldsymbol{\Pi}$ could win the game $\operatorname{EF}_{\kappa}(\mathcal{A}, \mathcal{B})$ when $\mathbf{I}$ uses $\tau$ : She plays according to the isomorphism $f$. Note that the first move of $\mathbf{I} \tau(\varnothing)$ is in $Y \cup Z$, as are all the subsequent moves, which is a contradiction.

On the other hand, assume that $\sigma$ is a winning strategy for $\boldsymbol{\Pi}$ without loss of generality in $\operatorname{EF}_{\kappa}^{*}(\mathcal{A}, \mathcal{B})$. If it were $X \cap \mathcal{A} \neq X \cap \mathcal{B}$, then $\mathbf{I}$ would win by enumerating all of $X$. 
By using the new Definition 2.10, previous lemmas and Theorem 2.11, it is not difficult to see the following (recall from (*) that $S=\{X \subset A \cup B|| X \mid \leqslant \kappa, X \cap \mathcal{A} \cong$ $X \cap \mathcal{B}\})$.

2.14. Theorem. If $S$ (resp. $[A \cup B]^{<\kappa^{+}} \backslash S$ ) contains a $\kappa$-cub set, then $\boldsymbol{\Pi}$ (resp. I) has a winning strategy in $\operatorname{EF}_{\kappa}^{*}(\mathcal{A}, \mathcal{B})$. If $\kappa^{<\kappa}=\kappa$, then the converse is also true: If $\boldsymbol{\Pi}$ (resp. I) wins the game $\operatorname{EF}_{\kappa}^{*}(\mathcal{A}, \mathcal{B})$, then $S$ (resp. $[A \cup B]^{<\kappa^{+}} \backslash S$ ) contains a $\kappa$-cub set.

Proof. Assume that $S$ contains a cub set $C$. Then player $\boldsymbol{\Pi}$ can pick an increasing sequence of elements of $C$ when playing $\operatorname{EF}_{\kappa}^{\kappa, \kappa}(\mathcal{A}, \mathcal{B})$, which leads to a win. Assume $\kappa^{<\kappa}=\kappa$ and that player $\mathbb{\Pi}$ has a winning strategy $\sigma$ in $\operatorname{EF}_{\kappa}^{*}(\mathcal{A} . \mathcal{B})$. Then $C=\{X \subset$ $A \cup B \mid X$ is closed under $\sigma\}$ is a $\kappa$-cub set by Lemma 2.12. Moreover, if $X \in C$, then $X \in S$ by Lemma 2.13 .

2.15. Corollary. If $\mathbf{I}$ (resp. II) does not have a winning strategy in $\operatorname{EF}_{\kappa}^{*}(\mathcal{A}, \mathcal{B})$, then $S$ (resp. $[A \cup B]^{<\kappa^{+}} \backslash S$ ) is $\kappa$-stationary (intersects all $\kappa$-cub sets).

\section{Similarity of $\mathrm{EF}_{\kappa} \mathrm{AND}^{\mathrm{E}} \mathrm{FF}_{\kappa}^{*}$}

Since the weak game is easier for the second player, the implications which are shown in Figure 1 are immediately verified.

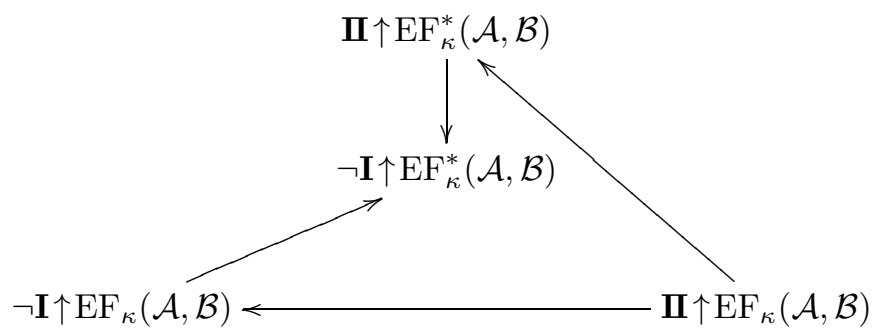

Figure 1. Implications that follow directly from the definitions of the games.

One more implication can be proved under $\kappa^{<\kappa}=\kappa$ :

3.1. Theorem. Let $\mathcal{A}$ and $\mathcal{B}$ be any structure and $\kappa$ a cardinal such that $\kappa^{<\kappa}=\kappa$. Then $\mathbf{I} \uparrow \mathrm{EF}_{\kappa}(\mathcal{A}, \mathcal{B}) \Rightarrow \mathbf{I} \uparrow \mathrm{EF}_{\kappa}^{*}(\mathcal{A}, \mathcal{B})$.

For later needs we shall prove a slightly more general result:

3.2. Theorem. Let $\mathcal{A}$ and $\mathcal{B}$ be any structure $\kappa$ a cardinal and $\alpha$ an ordinal satisfying $\kappa^{<\alpha}=\left|\bigcup_{\beta<\alpha} \kappa^{\beta}\right|=\kappa$. Then $\mathbf{I} \uparrow \mathrm{EF}_{\alpha}(\mathcal{A}, \mathcal{B}) \Rightarrow \mathbf{I} \uparrow \mathrm{EF}_{\kappa}^{*}(\mathcal{A}, \mathcal{B})$.

Proof. Assume that $\tau:(A \cup B)^{<\alpha} \rightarrow(A \cup B)$ is the winning strategy for player $\mathbf{I}$ in $\operatorname{EF}_{\alpha}(\mathcal{A}, \mathcal{B})$. We now claim that the set

$$
W=\left\{X \in[A \cup B]^{<\kappa^{+}} \mid X \text { is closed under } \tau \text { and } \tau(\varnothing) \in X\right\} \subset[A \cup B]^{\kappa^{+}}
$$

is $\kappa$-cub by Lemma 2.12, and for any $X \in W$ we have $X \cap \mathcal{A} \neq X \cap \mathcal{B}$ by Lemma 2.13. Thus $W$ is a $\kappa$-cub outside $S$.

Now by Theorem 2.14 $\mathbf{I}$ has a winning strategy in the game $\operatorname{EF}_{\kappa}^{*}(\mathcal{A}, \mathcal{B})$, and so also in the game $\mathrm{EF}_{\kappa}^{\circ}(\mathcal{A}, \mathcal{B})$. 
3.3. Corollary. If $\kappa$ satisfies $\kappa^{<\kappa}=\kappa$ and $\operatorname{EF}_{\kappa}(\mathcal{A}, \mathcal{B})$ is determined, then $\operatorname{EF}_{\kappa}^{*}(\mathcal{A}, \mathcal{B})$ as well as $\mathrm{EF}_{\kappa}^{\circ}(\mathcal{A}, \mathcal{B})$ are determined and

$$
\mathcal{A} \sim_{\kappa} \mathcal{B} \Longleftrightarrow \mathcal{A} \sim_{\kappa}^{\circ} \mathcal{B} \Longleftrightarrow \mathcal{A} \sim_{\kappa}^{*} \mathcal{B}
$$

Proof. When the EF-game is determined, we can add the implication $\neg \mathbf{I} \uparrow \mathrm{EF}_{\kappa}(\mathcal{A}, \mathcal{B})$ $\rightarrow \Pi \uparrow \mathrm{EF}_{\kappa}(\mathcal{A}, \mathcal{B})$ to the diagram of Figure 1 , and by Theorem 3.2 we can add the implication $\neg \mathbf{I} \uparrow \operatorname{EF}_{\kappa}^{*}(\mathcal{A}, \mathcal{B}) \rightarrow \neg \mathbf{I} \uparrow \operatorname{EF}_{\kappa}(\mathcal{A}, \mathcal{B})$. After completing all implications which follow by combining the existing ones, we obtain that all are equivalent:

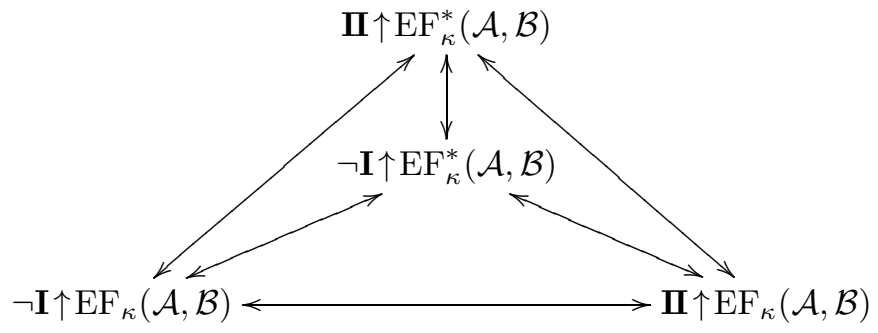

\section{Countable games}

The shortest infinite game $\mathrm{EF}_{\omega}^{*}$. Let $S=\{X \subset A \cup B \mid X \cap \mathcal{A} \cong X \cap$ $\mathcal{B}$ and $|X| \leqslant \omega\} \subset[A \cup B]^{<\omega_{1}}$ for some structures $\mathcal{A}$ and $\mathcal{B}$. Recall that $\mathcal{A} \equiv_{\infty} \boldsymbol{B}$ means that for all $\varphi \in L_{\infty \omega}, \mathcal{A} \models \varphi \Longleftrightarrow \mathcal{B} \models \varphi$. It was proved in Kue] (Theorem 3.5) that

(a) $\mathcal{A} \equiv_{\infty \omega} \mathcal{B} \Longleftrightarrow S$ contains a cub-set,

(b) $\mathcal{A} \neq_{\infty \omega} \mathcal{B} \Longleftrightarrow[A \cup B]^{<\omega_{1}} \backslash S$ contains a cub-set.

This can be reformulated by Theorem 2.14 as follows:

$\left(\mathrm{a}^{\prime}\right) \mathcal{A} \equiv_{\infty \omega} \mathcal{B} \Longleftrightarrow \Pi \uparrow \mathrm{EF}_{\omega}^{*}(\mathcal{A}, \mathcal{B})$,

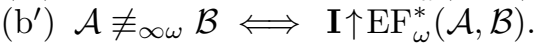

4.1. Theorem. The games $\operatorname{EF}_{\omega}^{\circ}(\mathcal{A}, \mathcal{B})$ and $\operatorname{EF}_{\omega}^{*}(\mathcal{A}, \mathcal{B})$ are determined for every $\mathcal{A}$ and $\mathcal{B}$, and

$$
\mathcal{A} \sim_{\omega} \mathcal{B} \Longleftrightarrow \mathcal{A} \sim_{\omega}^{\circ} \mathcal{B} \Longleftrightarrow \mathcal{A} \sim_{\omega}^{*} \mathcal{B} .
$$

Proof. It is well known that $\mathrm{EF}_{\omega}$ is determined, because it is a closed game. Because $\omega^{<\omega}=\omega$, we can apply Corollary 3.3

Remark. One can also obtain Theorem 4.1 also from $\left(\mathrm{a}^{\prime}\right),\left(\mathrm{b}^{\prime}\right)$ and the characterizations of $L_{\infty}$-equivalence by Karp:

$$
\begin{aligned}
& \left(\mathrm{a}^{\prime \prime}\right) \mathcal{A} \equiv_{\infty \omega} \mathcal{B} \Longleftrightarrow \mathbf{\Pi} \uparrow \operatorname{EF}_{\omega}(\mathcal{A}, \mathcal{B}), \\
& \left(\mathrm{b}^{\prime \prime}\right) \mathcal{A} \not \equiv_{\infty \omega} \mathcal{B} \Longleftrightarrow \mathbf{I} \uparrow \operatorname{EF}_{\omega}(\mathcal{A}, \mathcal{B}) .
\end{aligned}
$$

Counterexamples for game length $\alpha, \omega<\alpha<\omega_{1}$. As mentioned, the result of Theorem 4.1 does not work for finite ordinals (Example 2.8) and it does not generally extend, for example, to ordinals $\omega<\alpha<\omega_{1}$ either.

4.2. Example. Let $A=B=\omega_{1}, R$ be a unary relation satisfying $R^{\mathcal{A}}=\omega$ and $R^{\mathcal{B}}=\omega_{1} \backslash \omega$. Now clearly $\mathcal{A} \sim_{\omega} \mathcal{B}$. Also if $\mathbf{I}$ fills the set $\omega \subset A$ during the first $\omega$ moves, the second player loses the ordinary EF game on the next move, i.e. $\mathbf{I} \uparrow \operatorname{EF}_{\omega+1}(\mathcal{A}, \mathcal{B})$. But $\boldsymbol{\Pi}$ survives in the weak game. She survives as long as the 
length of the game is countable, because the only thing she has to do is to choose the same amount of points with properties $R$ and $\neg R$ as $\mathbf{I}$ does.

4.3. Example. Consider the structures constructed in NadSta: For $B \subset \omega_{1}$ let

$$
\Phi(B)=\bigcup_{\alpha<\omega_{1}}\{\alpha\} \times \tau_{\alpha},
$$

where $\tau_{\alpha}=1+\mathbb{Q}$ if $\alpha \in B$ and $\tau_{\alpha}=\mathbb{Q}$ if $\alpha \notin B$. The order on $\Phi$ is lexicographical; that is, $(\alpha, q)<(\beta, p)$ if $\alpha<\beta$ or $\alpha=\beta$ and $q<p$. We now set $\mathcal{A}=\Phi(\varnothing)$ and $\mathcal{B}=\Phi\left(\omega_{1} \backslash \omega\right)$. The game $\operatorname{EF}_{\omega+2}(\mathcal{A}, \mathcal{B})$ is a win for $\mathbf{I}$, which implies the same for $\operatorname{EF}_{\omega+n}(\mathcal{A}, \mathcal{B})$, where $n \geqslant 2$.

On the other hand it is easy to see that $\Pi \uparrow \mathrm{EF}_{\omega+n}^{*}(\mathcal{A}, \mathcal{B})$.

The following example is given to manifest that player $\boldsymbol{\Pi}$ can lose a shorter game and win a longer one.

4.4. Example. Let $\mathcal{A}=\langle\mathbb{R},<\rangle$ be the real numbers with the usual ordering and $\mathcal{B}$ with domain $B=\mathbb{R} \times \omega_{1}$ and lexicographical ordering $((x, \alpha)<(y, \beta) \Longleftrightarrow \alpha<$ $\beta \vee(\alpha=\beta \wedge x<y))$. These are dense linear orderings and are $\mathrm{EF}_{\omega}$-equivalent as a simple back-and-forth argument shows; thus $\Pi \uparrow \mathrm{EF}_{\omega}^{*}(\mathcal{A}, \mathcal{B})$. However $\mathbf{I} \uparrow$ $\operatorname{EF}_{\omega+1}^{*}(\mathcal{A}, \mathcal{B})$ : He can play so that an unbounded set of $\mathcal{A}$ is chosen during the first $\omega$ moves. But since any countable subset of $\mathcal{B}$ is bounded, he can play an upper bound on the last move, $\omega+1$. But when the length of the game is increased to $\omega+\omega$, player $\boldsymbol{I}$ wins again by picking countable elementarily equivalent substructures. In fact $\mathbf{I} \uparrow \operatorname{EF}_{\alpha}^{*}(\mathcal{A}, \mathcal{B})$ for successors $\omega<\alpha<\omega_{1}$ and $\Pi \uparrow \mathrm{EF}_{\alpha}^{*}(\mathcal{A}, \mathcal{B})$ for limit ordinals $\omega \leqslant \alpha<\omega_{1}$.

\section{LONGER GAMES}

In this section we will show that it is consistent with ZFC that

- $\mathrm{EF}_{\omega_{1}}$ and $\mathrm{EF}_{\omega_{1}}^{*}$ are equivalent on structures of cardinality $\leqslant \aleph_{2}$ and are both determined, provided that the existence of a certain large cardinal is consistent.

- There are structures $\mathcal{A}$ and $\mathcal{B}$ such that $|A|=|B|=\aleph_{2}$, and $\mathcal{A} \varkappa_{\omega_{1}} \mathcal{B}$ but $\mathcal{A} \sim_{\omega_{1}}^{*} \mathcal{B}$.

- There are structures $\mathcal{A}, \mathcal{B}, \mathcal{A}^{\prime}$ and $\mathcal{B}^{\prime}$ such that $|A|=|B|=\left|\mathcal{A}^{\prime}\right|=\left|\mathcal{B}^{\prime}\right|=\aleph_{2}$, and $\mathcal{A} \varkappa_{\omega_{1}} \mathcal{B}$ but $\mathcal{A} \sim_{\omega_{1}}^{\circ} \mathcal{B}$ and $\mathcal{A}^{\prime} \chi_{\omega_{1}}^{\circ} \mathcal{B}^{\prime}$ but $\mathcal{A}^{\prime} \sim_{\omega_{1}}^{*} \mathcal{B}^{\prime}$

- There are structures $\mathcal{A}$ and $\mathcal{B}$ such that $|A|=|B|=\aleph_{2}$ and $\operatorname{EF}_{\omega_{1}}^{*}(\mathcal{A}, \mathcal{B})$ is not determined.

- There are structures $\mathcal{A}$ and $\mathcal{B}$ and cardinals $\alpha_{0}<\beta_{0}<\alpha_{1}<\beta_{1}<\cdots$ such that $|A|=|B|=\aleph_{\omega \cdot \omega+1}$, for all $n<\omega, \alpha_{n}$ is regular and $\beta_{n}$ is singular, and $\mathcal{A} \chi_{\alpha_{n}}^{*} \mathcal{B}$ but $\mathcal{A} \sim_{\beta_{n}}^{*} \mathcal{B}$ for all $n<\omega$.

Finally, in ZFC we prove that there are structures $\mathcal{A}$ and $\mathcal{B}$ (of course larger than $\left.\aleph_{2}\right)$ such that $\mathrm{EF}_{\omega_{1}}^{*}(\mathcal{A}, \mathcal{B})$ is non-determined.

All games can be determined on structures of size $\aleph_{2}$. In [HytSheVää] the following was proved (Corollary 13):

5.1. Theorem. It is consistent, relative to the consistency of a weakly compact cardinal, that $C H$ and the game $\operatorname{EF}_{\omega_{1}}(\mathcal{A}, \mathcal{B})$ is determined for all $\mathcal{A}$ and $\mathcal{B}$ of cardinality $\leqslant \aleph_{2}$. 
5.2. Corollary. Assuming the consistency of a weakly compact cardinal, it is consistent that $\mathrm{CH}$ holds and the games $\mathrm{EF}_{\omega_{1}}$ and $\mathrm{EF}_{\omega_{1}}^{*}$ are equivalent and that both games are determined on all structures of cardinality $\aleph_{2}$.

Proof. By Theorem 5.1 and $\mathrm{CH}$ we can use Corollary 3.3 to obtain the result.

$\mathcal{A} \sim_{\kappa}^{*} \mathcal{B} \nRightarrow \mathcal{A} \sim_{\kappa} \mathcal{B}$ on structures of size $\kappa^{+}$. Let us fix an uncountable regular cardinal $\kappa$. We shall construct groups $\mathcal{F}$ and $\mathcal{G}$ such that $\mathrm{EF}_{\kappa}(\mathcal{F}, \mathcal{G})$ is nondetermined. In fact $\mathcal{F}$ is the free abelian group of cardinality $\kappa^{+}$and $\mathcal{G}$ will be an almost free abelian group of the same cardinality constructed using the combinatorial principle $\square_{\kappa}$. This construction was done in MekSheVää] in the case $\kappa=\omega_{1}$ and is almost identical. The proof that $\operatorname{EF}_{\kappa}(\mathcal{F}, \mathcal{G})$ is non-determined is exactly the same as is the proof for $\kappa=\omega_{1}$ in [MekSheVää]. Formally in this section, these groups will be models of a relational vocabulary.

5.3. Definition. The statement $\square_{\kappa}$ says that there exists a sequence $\left\langle C_{\alpha}\right| \alpha<$ $\left.\kappa^{+}, \bigcup \alpha=\alpha\right\rangle$ of sets with the properties

(i) $C_{\alpha}$ is a closed and unbounded subset of $\alpha$.

(ii) If $\operatorname{cf}(\alpha)<\kappa$, then $\left|C_{\alpha}\right|<\kappa$.

(iii) If $\gamma$ is a limit point of $C_{\alpha}$, then $C_{\gamma}=C_{\alpha} \cap \gamma$.

For the proof of the next theorem the reader is referred to [Jech] or to the primary source of this result by Jensen [Jen.

5.4. Theorem. If $V=L$, then $\square_{\kappa}$ holds.

This square principle, $\square_{\kappa}$, implies the existence of a non-reflecting stationary set $E$ on $\kappa^{+}$which we will use to construct our groups. Recall the notation $S_{\omega}^{\kappa^{+}}=$ $\left\{\alpha<\kappa^{+} \mid \operatorname{cf}(\alpha)=\omega\right\}$.

5.5. Lemma. Assume $\square_{\kappa}$. Then there exists an $\omega$-stationary set $E \subset S_{\omega}^{\kappa^{+}}$such that for every ordinal $\gamma<\kappa^{+}$of cofinality $\kappa$, the set $E \cap \gamma$ is non-stationary on $\gamma$.

Proof. This is standard and can be found, for example, in [Jech].

Now we are ready to construct the groups we talked about at the beginning of this section. We shall use some well-known facts about free abelian groups, direct products, etc. As we already noted, in this section groups will be models of a relational vocabulary. Substructures are not necessarily groups.

As both $\square_{\kappa}$ and GCH hold if $V=L$, the use of GCH makes no contradiction. The first group $\mathcal{F}$ will be the free abelian group generated by $\kappa^{+}$:

$$
\mathcal{F}=\bigoplus_{i<\kappa^{+}} \mathbb{Z}
$$

Another group will be a so-called almost free abelian group. The idea is that an almost free group $G$ is the union $G=\bigcup_{i<\kappa^{+}} G_{i}$ of its subgroups $G_{i}$ satisfying:

- Each $G_{i}$ is free.

- $G_{i} \subset G_{j}$ whenever $i<j$.

- $G$ is not free.

5.6. Definition. A subgroup $S$ of an abelian group $G$ (write it additively) is pure if for all $x \in S(\exists y \in G(n y=x)) \rightarrow(\exists y \in S(n y=x))$. That is, if $x \in S$ is divisible in $G$, it has to be divisible in $S$. 
Let $\mathbb{Z}^{\kappa^{+}}$stand for the direct product $\Pi_{\alpha<\kappa^{+}} \mathbb{Z}$ of $\kappa^{+}$copies of integers. By $x_{\gamma}$ we shall denote the element of $\mathbb{Z}^{\kappa^{+}}$which is zero on coordinates $\neq \gamma$ and 1 on the coordinate $\gamma$.

For each $\delta \in E$ (of Lemma 5.5) let us fix an increasing cofinal function $\eta_{\delta}: \omega \rightarrow \delta$ so that $\eta_{\delta}[\omega] \cap E=\varnothing$ (for instance take successor ordinals only). Define

$$
z_{\delta}=\sum_{n=0}^{\infty} 2^{n} x_{\eta_{\delta}(n)} \in \mathbb{Z}^{\kappa^{+}}
$$

For each $\alpha \leqslant \kappa^{+}$let $\mathcal{G}_{\alpha}$ be the smallest pure subgroup of $\mathbb{Z}^{\kappa^{+}}$which contains the set $\left\{x_{\gamma} \mid \gamma<\alpha\right\} \cup\left\{z_{\delta} \mid \delta \in E \cap \alpha\right\}$. We set $\mathcal{G}=\mathcal{G}_{\kappa^{+}}$. Also let $\mathcal{F}_{\alpha}$ be the free abelian group generated by $\left\{x_{\gamma} \mid \gamma<\alpha\right\}$ and set $\mathcal{F}=\mathcal{F}_{\kappa^{+}}$. We shall denote by $\left\langle y_{\alpha} \mid \alpha<\beta\right\rangle$ the group generated by the set $\left\{y_{\alpha} \mid \alpha<\beta\right\}$.

The proof of the following lemma and the following theorem are exactly as in MekSheVää, with $\omega_{1}$ changed to $\kappa$.

5.7. Lemma. For each $\alpha<\kappa^{+}$the group $\mathcal{G}_{\alpha}$ is free, and if $\beta \in \alpha \backslash E$, then any free basis of $\mathcal{G}_{\beta}$ can be extended to a free basis of $\mathcal{G}_{\alpha}$.

5.8. Theorem. If $\square_{\kappa}$ and $G C H$, in particular if $V=L$, then $\operatorname{EF}_{\kappa}(\mathcal{F}, \mathcal{G})$ is not determined.

Remark. GCH can be avoided; see MekSheVää].

Proof. (Sketch.) Player I does not win: The set $S=\{\alpha \mid E \cap \alpha$ is non-stationary $\}$ is stationary. Given a strategy $\tau$ of $\mathbf{I}$, the set $\left\{\alpha \mid \mathcal{F}_{\alpha} \cup \mathcal{G}_{\alpha}\right.$ is closed undet $\left.\tau\right\}$ intersects $S$ being cub and there is an isomorphism $\mathcal{F}_{\alpha} \cong \mathcal{G}_{\alpha}$. So $\boldsymbol{I I}$ just follows the isomorphism.

Player $\Pi$ doe not win: Assume that $\sigma$ is a winning strategy for player $\Pi$. Player I takes such an $\alpha \in E$ that $\mathcal{F}_{\alpha} \cup \mathcal{G}_{\alpha}$ is closed under the first $\omega$ moves of $\Pi$. In those first $\omega$ moves player I picks $\left\{x_{\eta_{\alpha}(n)} \mid n<\omega\right\}$ and a direct summand of $\mathcal{F}_{\alpha}$. Let $J$ be the set played so far in $\mathcal{G}_{\alpha}$. In the next $\omega$ moves I picks the smallest pure subgroup of $\mathcal{G}$ containing $J \cup\left\{z_{\delta}\right\}$. Denote it by $A$. Now $A / J$ is not a free group, but the corresponding structure $K / I$ in $\mathcal{F}$ ( $I$ are the first $\omega$ moves in $\mathcal{F}$, and $K$ are the first $\omega+\omega$ moves) is free. In the ordinary EF-game the isomorphism has to respect the order of moves, hence a contradiction.

\subsection{Theorem. Player $\Pi$ wins $\operatorname{EF}_{\kappa}^{*}(\mathcal{F}, \mathcal{G})$.}

Proof. Recall Theorem 2.11. In the game $\mathrm{EF}_{\kappa}^{*}, \kappa$ player $\Pi$ can choose on each move the set $\mathcal{F}_{\beta} \cup \mathcal{G}_{\beta}$, where $\beta$ is such that all elements played before this move are in $\mathcal{F}_{\beta} \cup \mathcal{G}_{\beta}$. Eventually substructures $\mathcal{F}_{\alpha}$ and $\mathcal{G}_{\alpha}$ are picked at the end of the game. By Lemma 5.7 they are isomorphic.

Remark. In the article HytSheVää, the authors construct linear orders $I$ and $J$ so that $\operatorname{EF}_{\omega_{1}}(I, J)$ is non-determined using a weaker assumption than we did for the construction of $\mathcal{F}$ and $\mathcal{G}$. However, it seems to the authors that there is no reason why player $\Pi$ would win the game $\operatorname{EF}_{\omega_{1}}^{*}(I, J)$. Also the theory of abelian groups is more interesting in this context, because it is stable. 
$\mathcal{A} \sim_{\kappa}^{*} \mathcal{B}$ does not imply $\mathcal{A} \sim_{\kappa}^{\circ} \mathcal{B}$, and $\mathcal{A} \sim_{\kappa}^{\circ} \mathcal{B}$ does not imply $\mathcal{A} \sim_{\kappa} \mathcal{B}$ for $\mathcal{A}$ and $\mathcal{B}$ of cardinality $\kappa^{+}$. Here we shall show that all these games can be different on structures of size $\kappa^{+}$. In all parts of this section $\kappa$ is a regular uncountable cardinal.

To prove that $\mathrm{EF}_{\kappa}^{\circ}$ is different from $\mathrm{EF}_{\kappa}$, we use a vocabulary with function symbols.

$\mathcal{A} \sim_{\kappa}^{\circ} \mathcal{B}$ does not imply $\mathcal{A} \sim_{\kappa} \mathcal{B}$. In this section we will use groups as models of a functional vocabulary. Thus instead of relation $+_{R}$ we have function symbols + and - whose interpretations satisfy $+(x, y)=z \Longleftrightarrow(x, y, z) \in+_{R}$, etc.

5.10. Theorem $(\mathrm{GCH})$. Let $\mathcal{F}^{\prime}$ and $\mathcal{G}^{\prime}$ be the groups constructed in the previous section presented with function symbols,+- . Then $\mathrm{EF}_{\kappa}\left(\mathcal{F}^{\prime}, \mathcal{G}^{\prime}\right)$ is non-determined.

Proof. The same reason as to why $\operatorname{EF}_{\kappa}(\mathcal{F}, \mathcal{G})$ is non-determined.

5.11. Theorem $(\mathrm{GCH})$. Let $\mathcal{F}^{\prime}$ and $\mathcal{G}^{\prime}$ be the groups constructed in the previous paragraph presented with function symbols + , - . Then player $\Pi$ wins $\operatorname{EF}_{\kappa}^{\circ}\left(\mathcal{F}^{\prime}, \mathcal{G}^{\prime}\right)$.

Proof. Note that now any substructure is a subgroup. Let us provide a winning strategy for $\boldsymbol{I}$ by induction. Assume that on the move $\alpha$ the position of the game is such that the players have chosen $X \subset \mathcal{F}^{\prime}$ and $Y \subset \mathcal{G}^{\prime}$ and the subgroups $\langle X\rangle$ and $\langle Y\rangle$ are isomorphic. Assume that $\mathbf{I}$ next picks $x \in \mathcal{F}^{\prime}$. The dimension of a free abelian group is the cardinality of its basis. Note that it is unique, and in the case of abelian groups the dimension of a subgroup is always less than or equal to the dimension of the super-group. If

$$
\operatorname{dim}\langle X \cup\{x\}\rangle>\operatorname{dim}\langle X\rangle,
$$

then obviously

$$
\operatorname{dim}\langle X \cup\{x\}\rangle=\operatorname{dim}\langle X\rangle+1,
$$

wherefore let $\boldsymbol{I}$ pick an element $y \in \mathcal{G}^{\prime}$ such that

$$
\operatorname{dim}\langle Y \cup\{y\}\rangle=\operatorname{dim}\langle X \cup\{x\}\rangle
$$

(it is possible since $X$ and $Y$ are still subsets of $\operatorname{dom}\left(\mathcal{F}^{\prime}\right)$ and $\operatorname{dom}\left(\mathcal{G}^{\prime}\right)$ of size $\kappa$, while $\left.\left|\operatorname{dom}\left(\mathcal{F}^{\prime}\right)\right|=\left|\operatorname{dom}\left(\mathcal{G}^{\prime}\right)\right|=\kappa^{+}\right)$. On the other hand, if $x$ is such that $\operatorname{dim}\langle X \cup\{x\}\rangle=\operatorname{dim}\langle X\rangle$, then we have three cases:

C1: $\operatorname{dim}\langle X\rangle\langle\omega$. She has to pick an element which is already in $\langle Y\rangle$.

C2: $\operatorname{dim}\langle X\rangle \geqslant \omega$ and $x \in\langle X\rangle$. She has to pick an element which is already in $\langle Y\rangle$.

C3: $\operatorname{dim}\langle X\rangle \geqslant \omega$ and $x \notin\langle X\rangle$. She has to pick an element which is in $\mathcal{G}^{\prime} \backslash\langle Y\rangle$. If I picks an element from $\mathcal{G}^{\prime}$ instead of $\mathcal{F}^{\prime}$, the reasoning for player $\boldsymbol{\Pi}$ would be exactly the same, with the structures switched.

This strategy guarantees that at each move the groups generated by the played sequences remain isomorphic, and simultaneously it guarantees that if player $\mathbf{I}$ picks at the end of the game $\kappa$ points from one of the structures, then the same amount is picked from the other one and moreover the chosen groups are isomorphic, because their sets of generators are of the same cardinality.

5.12. Corollary. In the class of structures of size $\kappa^{+}, \mathcal{A} \sim_{\kappa}^{\circ} \mathcal{B}$ does not imply $\mathcal{A} \sim_{\kappa} \mathcal{B}$. 
$\mathcal{A} \sim_{\kappa}^{*} \mathcal{B}$ does not imply $\mathcal{A} \sim_{\kappa}^{\circ} \mathcal{B}$. Let us consider two structures, $\mathcal{A}$ and $\mathcal{B}$, such that

- $\operatorname{EF}_{\kappa}(\mathcal{A}, \mathcal{B})$ is non-determined,

- $\amalg \uparrow \mathrm{EF}_{\kappa}^{*}(\mathcal{A}, \mathcal{B})$,

- $|\mathcal{A}|=|\mathcal{B}|=\kappa^{+}$.

Such structures $\mathcal{A}$ and $\mathcal{B}$ of cardinality $\kappa^{+}$exist by the previous section (the free and almost free abelian groups of cardinality $\kappa^{+}$).

Using these structures, we shall construct new structures $M(\mathcal{A})$ and $M(\mathcal{B})$ such that $\mathrm{EF}_{\kappa}^{\circ}(M(\mathcal{A}), M(\mathcal{B}))$ is non-determined but $\amalg \uparrow \mathrm{EF}_{\kappa}^{*}(M(\mathcal{A}), M(\mathcal{B}))$.

We have $|M(\mathcal{A})|=|M(\mathcal{B})|=2^{\kappa}$. If GCH holds, then $|M(\mathcal{A})|=|M(\mathcal{B})|=\kappa^{+}$.

5.13. Definition. Let $\mathcal{A}$ be an $L$-structure. Let

$$
L^{+}=L \cup\{<\} \cup\left\{P_{\alpha} \mid \alpha<\kappa, P_{\alpha} \text { is a unary relation symbol }\right\},
$$

where the new symbols are not in $L$. See the remark at the end of this section as to how to get rid of an infinite vocabulary. We define $M(\mathcal{A})$ to be the $L^{+}$-structure with the domain

$$
\operatorname{dom}(M(\mathcal{A}))=\{f: \alpha+1 \rightarrow A \mid \alpha<\kappa\},
$$

and if $f_{i} \in \operatorname{dom}(M(\mathcal{A})), i<n$, and $R$ is an $n$-place relation symbol of the vocabulary, we define

$$
\left(f_{0}, \ldots, f_{n-1}\right) \in R^{M(\mathcal{A})} \Longleftrightarrow\left(f_{0}\left(\alpha_{0}\right), \ldots, f_{n-1}\left(\alpha_{n-1}\right)\right) \in R^{\mathcal{A}},
$$

where $\alpha_{i}$ is the maximum of the domain of $f_{i}$. The partial order $f<g$ is defined for $f, g \in M(\mathcal{A})$ so that $f<^{M(\mathcal{A})} g$ if $f \subset g$; that is, $g\lceil\operatorname{dom}(f)=f$. The relations $P_{\alpha}$ are interpreted as $P_{\alpha}^{M(\mathcal{A})}=\{f \mid \operatorname{dom} f=\alpha+1\}$.

Note that if $\mathcal{A}$ and $\mathcal{B}$ are isomorphic, then $M(\mathcal{A})$ and $M(\mathcal{B})$ are isomorphic. Also if $\left(f_{i}\right)_{i<\alpha}$ is an increasing chain, then the reduction of the substructure $\left\{f_{i} \mid i<\right.$ $\alpha\} \subset M(\mathcal{A})$ to $L$ is isomorphic to the substructure $\left\{f_{i}\left(\max \left(\operatorname{dom}\left(f_{i}\right)\right)\right) \mid i<\alpha\right\} \subset$ $\mathcal{A}$. But if we have a chain $\left\{f_{i} \mid i<\alpha\right\}$ in $M(\mathcal{A})$ and another chain $\left\{g_{i} \mid i<\alpha\right\}$ in $M(\mathcal{B})$, then if there is an isomorphism $\left\{f_{i} \mid i<\alpha\right\} \rightarrow\left\{g_{i} \mid i<\alpha\right\}$, then it has to be order preserving.

Recall that $\mathcal{A}$ and $\mathcal{B}$ are chosen so that $\operatorname{EF}_{\kappa}(\mathcal{A}, \mathcal{B})$ is non-determined but $\Pi \uparrow$ $\mathrm{EF}_{\kappa}^{*}(\mathcal{A}, \mathcal{B})$. We now claim that player $\boldsymbol{I}$ does not win $\mathrm{EF}_{\kappa}^{\circ}(M(\mathcal{A}), M(\mathcal{B}))$.

5.14. Theorem. Player $\Pi$ does not have a winning strategy in $\mathrm{EF}_{\kappa}^{\circ}(M(\mathcal{A}), M(\mathcal{B}))$.

Proof. Let us make a counter assumption: $\sigma$ is a winning strategy for $\boldsymbol{\Pi}$ in

$$
\mathrm{EF}_{\kappa}^{\circ}(M(\mathcal{A}), M(\mathcal{B})) \text {. }
$$

We will find a strategy for $\mathbf{I}$ which beats this strategy, which is a contradiction.

Let us assume that players pick only increasing <-chains and at the move $\alpha$ they pick elements from $P_{\alpha}^{M(\mathcal{A})} \cup P_{\alpha}^{M(\mathcal{B})}$. Here there is no loss of generality, since if player $\mathbf{I}$ picks only such elements, then player $\boldsymbol{I}$ is forced to do the same.

Let us define $\sigma^{*}$ : A strategy for player $\Pi$ in $\operatorname{EF}_{\kappa}(\mathcal{A}, \mathcal{B})$ by

$$
\sigma^{*}\left(\left(x_{i}\right)_{i \leqslant \gamma}\right)=\operatorname{md}\left[\sigma\left(\left\langle f_{\alpha}\right\rangle_{\alpha \leqslant \gamma}\right)\right] \text {, where: }
$$

$\left(x_{i}\right)_{i \leqslant \gamma}$ is the sequence of the moves previously played by $\mathbf{I} ; f_{\alpha}$ is defined so that $\operatorname{dom} f_{\alpha}=\alpha+1, f_{\alpha}(\alpha)=x_{\alpha}$, and if $x_{\alpha} \in \mathcal{A}$, then for every $i<\alpha$ we have that $f_{\alpha}(i)$ is the $i$ th move from $\mathcal{A}$ (made by $\mathbf{I}$ or $\mathbf{I}$ ) and if $x_{\alpha} \in \mathcal{B}$, then $f_{\alpha}(i)$ is the $i$ th move from $\mathcal{B}$; and finally $\operatorname{md}[f]=f(\max \operatorname{dom}(f))$. 
By the assumption there is a strategy $\tau^{*}$ of player $\mathbf{I}$ in $\operatorname{EF}_{\kappa}(\mathcal{A}, \mathcal{B})$ which beats $\sigma^{*}$. Let us define the strategy for $\mathbf{I}$ in $\operatorname{EF}_{\kappa}^{\circ}(M(\mathcal{A}), M(\mathcal{B}))$ as follows. Assume that $\left(f_{i}\right)_{i<\gamma}$ is the sequence of functions $\in M(\mathcal{A}) \cup M(\mathcal{B})$ that player $\boldsymbol{I}$ had chosen in the $\gamma$ first moves. Let $\mathcal{C}$ stand for $\mathcal{A}$ if $\tau^{*}\left(\left(f_{i}(i)\right)_{i<\gamma}\right)$ is in $\mathcal{A}$ and for $\mathcal{B}$ if it is in $\mathcal{B}$. Then define

$\tau\left(\left(f_{i}\right)_{i<\gamma}\right)=\bigcup\{g \in M(\mathcal{C}) \mid g$ is played at some move $\beta<\gamma\} \cup\left\{\left(\gamma, \tau^{*}\left(\left(f_{i}(i)\right)_{i<\gamma}\right)\right)\right\}$.

Let us show that $\tau$ beats $\sigma$. Because $\tau^{*}$ beats $\sigma^{*}$, the function between the played sets which respects the moves is not an isomorphism. But there cannot be any other isomorphism as well, since an isomorphism should respect the order $<$ and the levels $P_{\alpha}$, so the only possible isomorphism would be the one induced by the order of the moves.

However, it is necessary for $\mathbf{I}$ to be able to choose from which structure to play:

5.15. Theorem (GCH). Player $\Pi$ has a winning strategy in $\mathrm{EF}_{\kappa}^{*}(M(\mathcal{A}), M(\mathcal{B}))$.

Proof. Again, the only thing we use about $\mathcal{A}$ and $\mathcal{B}$ is that $\operatorname{EF}_{\kappa}(\mathcal{A}, \mathcal{B})$ is nondetermined but $\Pi \uparrow \mathrm{EF}_{\kappa}^{*}(\mathcal{A}, \mathcal{B})$.

If $X \subset \mathcal{A} \cup \mathcal{B}$, let

$$
N(X)=\{f \in M(\mathcal{A}) \cup M(\mathcal{B}) \mid \operatorname{ran} f \subset X\},
$$

and if $Y \subset M(\mathcal{A}) \cup M(\mathcal{B})$, then

$$
N^{-1}(Y)=\{x \in \mathcal{A} \cup \mathcal{B} \mid x \in \operatorname{ran} f \text { for some } f \in Y\} .
$$

Realize that for all $X, X^{\prime} \subset \mathcal{A} \cup \mathcal{B}, Y, Y^{\prime} \subset M(\mathcal{A}) \cup M(\mathcal{B})$ we have

- $|X| \leqslant \kappa \Longleftrightarrow N(X) \leqslant \kappa$,

- $N\left(N^{-1}(Y)\right) \supset Y$

- $N^{-1}(N(X))=X$,

- $N(X \cap \mathcal{A})=N(X) \cap M(\mathcal{A})$ and $N(X \cap \mathcal{B})=N(X) \cap M(\mathcal{B})$,

- $X \cong X^{\prime} \Longleftrightarrow N(X) \cong N\left(X^{\prime}\right)$.

By Theorem 2.14 it is enough to show that there is a $\kappa$-cub set

$$
C \subset S=\{X \subset M(\mathcal{A}) \cup M(\mathcal{B})|X \cap M(\mathcal{A}) \cong X \cap M(\mathcal{B}),| X \mid \leqslant \kappa\} .
$$

We know that $S^{\prime}=\{X \subset \mathcal{A} \cup \mathcal{B}|X \cap \mathcal{A} \cong X \cap \mathcal{B}| X \mid, \leqslant \kappa\}$ contains a cub set. Let it be denoted by $C^{\prime}$. We claim that the set

$$
C=\left\{Y \subset M(\mathcal{A}) \cup M(\mathcal{B}) \mid Y=N(X), X \in C^{\prime}\right\}
$$

is cub and contained in $S$. Because $X \cong Y \Rightarrow N(X) \cong N(Y)$, it is clear that $C \subset S$. Let us show that it is cub.

Let $Y \in C$. Then there is $X \in C^{\prime}$ such that $X \supset N^{-1}(Y)$. Then $N(X) \supset$ $N\left(N^{-1}(Y)\right) \supset Y$. On the other hand, because $X \cap \mathcal{A} \cong X \cap \mathcal{B}$, we get

$$
N(X) \cap M(\mathcal{A})=N(X \cap \mathcal{A}) \cong N(X \cap \mathcal{B})=N(X) \cap M(\mathcal{B}) .
$$

Thus $C$ is unbounded.

Assume that $\left(Y_{i}\right)_{i<\kappa}=\left(N\left(X_{i}\right)\right)_{i<\kappa}$ is an increasing chain in $C$. Then $X_{i}$ is in fact an increasing chain in $C^{\prime}$. Thus we know $\bigcup_{i<\kappa} X_{i} \in C^{\prime}$. But then $N\left(\bigcup_{i<\kappa} X_{i}\right) \in C$, and it easy to see that

$$
N\left(\bigcup_{i<\kappa} X_{i}\right)=\bigcup_{i<\kappa} N\left(X_{i}\right) .
$$


It is easy to see because the functions always have a domain of cardinality less than $\kappa$, so if $f \in N\left(\bigcup_{i<\kappa} X_{i}\right)$, then surely $f \in N\left(\bigcup_{i<\alpha} X_{i}\right)$ for some $\alpha<\kappa$, and since the chain is increasing this implies $f \in X_{\alpha}$.

5.16. Corollary. In the class of structures of size $\kappa^{+}, \mathcal{A} \sim_{\kappa}^{*} \mathcal{B}$ does not imply $\mathcal{A} \sim_{\kappa}^{\circ} \mathcal{B}$

Remark. We used an uncountable vocabulary $L^{+}$as the vocabulary of $M(A)$ and $M(B)$ because we wanted to fix the levels of the <-tree. However, we can do that by using only a finite extension of the vocabulary, assuming that $\kappa$ is a successor cardinal. By Theorem 0.4 of Chapter VIII of [She, if $T$ is not a superstable theory, then there are models $\mathcal{A}_{i}$ of $T, i<2^{\kappa}$, for which we have $\left|\mathcal{A}_{i}\right|=\kappa$ for all $i$, and for all distinct indices $i, j$ the model $\mathcal{A}_{i}$ cannot be elementarily embedded in $\mathcal{A}_{j}$.

Because the theory of dense linear orderings without end points is unstable and has quantifier elimination, there exist $2^{\kappa}$ (we need only $\kappa$ ) pairwise non-embeddable to each other linear orderings of cardinality $\kappa$. Let $\left\{Q_{i} \mid i<\kappa\right\}$ be a collection of such linear orderings. Let $L, \mathcal{A}$ and $\mathcal{B}$ be as at the beginning of this section and define $L^{+}=L \cup\left\{<,<^{*}, R\right\}$, where the new symbols are binary relations. Let $M(\mathcal{A})$ and $M(\mathcal{B})$ be the structures defined in this section except without the relations $P_{\alpha}$. Let us now define $M^{\prime}(\mathcal{A})\left(M^{\prime}(B)\right.$ is similar). The domain is the disjoint union

$$
\operatorname{dom}\left(M^{\prime}(\mathcal{A})\right)=\operatorname{dom}(M(\mathcal{A})) \cup \bigcup\left\{Q_{i} \mid i<\kappa\right\} .
$$

The symbol $<^{*}$ is interpreted as the ordering of the linear orderings $Q_{i}$ and $R$ is interpreted as follows:

$$
(f, q) \in R \Longleftrightarrow f \in \operatorname{dom}(M(\mathcal{A})) \wedge \operatorname{dom}(f)=i+1 \wedge q \in Q_{i} ;
$$

i.e. we fix the $(i+1)$ st level by the linear ordering $Q_{i}$. Now if at any move player $\boldsymbol{\Pi}$ plays at a different level than $\mathbf{I}$, then he will play the corresponding linear ordering and $\Pi$ will not be able to embed it to any other than the same one, thus losing the game.

$\mathrm{EF}_{\omega_{1}}^{*}$ can be non-determined on structures of size $\aleph_{2}$. Recall that, by Corollary 2.15] in order to construct $\mathcal{A}$ and $\mathcal{B}$ such that $\operatorname{EF}_{\omega_{1}}^{*}(\mathcal{A}, \mathcal{B})$ is non-determined, we have to find such models $\mathcal{A}$ and $\mathcal{B}$ that the set $\{X \subset A \cup B \mid X \cap \mathcal{A} \cong X \cap \mathcal{B}\}$ is at least $\omega_{1}$-bistationary; i.e. a stationary set whose complement is also stationary (if $\mathrm{CH}$, then it is enough).

5.17. Definition. Let $\omega \leqslant \lambda \leqslant \alpha<\mu$ be such that $\lambda$ and $\mu$ are regular cardinals and $\alpha$ is an ordinal. Then let $S \subset \mu$. The cub-game $G_{\lambda}^{\alpha}(S)$ is the following game played by players $\mathbf{I}$ and $\boldsymbol{I}$. On the move $\gamma<\alpha$ first player $\mathbf{I}$ picks $x_{\gamma} \in \mu$ so that $x_{\gamma}$ is greater than any element played so far in the game, and then player $\boldsymbol{\Pi}$ chooses $y_{\gamma} \in S$ so that $y_{\gamma}>x_{\gamma}$. Finally, sequences $\left(x_{\gamma}\right)_{\gamma<\alpha}$ and $\left(y_{\gamma}\right)_{\gamma<\alpha}$ are formed. Player II wins if

(1) she has played according to the rules and

(2) $\operatorname{cl}_{\lambda}\left\{y_{\gamma} \mid \gamma<\alpha\right\} \subset S$,

where $\operatorname{cl}_{\lambda} B$ is the smallest $\lambda$-closed set which contains $B$.

For more on these games, see HytSheTuu and Hyt.

Let us consider the following construction. Let $\mu$ be an uncountable cardinal and $S \subset S_{\omega}^{\mu}$. In the following $\mu \times \omega$ is equipped with reversed lexicographical order 
and $\mathrm{pr}_{\mu}$ and $\mathrm{pr}_{\omega}$ are projections, respectively, onto $\mu$ and $\omega$. Then let

$$
A(\mu, S)=\{f: \alpha+1 \rightarrow \mu \times \omega \mid \alpha<\mu,
$$

$f$ is strictly increasing, according to the reversed lexicographical order,

for each $n<\omega$ the set $\operatorname{pr}_{\mu}[\operatorname{ran}(f) \cap(\mu \times\{n\})]$

is $\omega$-closed in $\mu$ and is contained in $S\}$

and

$$
\begin{aligned}
B(\mu, S)=\{ & f: \alpha+1 \rightarrow \mu \times \omega \mid \alpha<\mu, \\
& f \text { is strictly increasing, } \\
& \text { for each } n<\omega \text { the set } \operatorname{pr}_{\mu}[\operatorname{ran}(f) \cap(\mu \times\{n\})]
\end{aligned}
$$

is $\omega$-closed as a subset of $\mu$, and if $n>0$, then is contained in $S\}$.

The structures $\mathcal{A}(\mu, S)$ and $\mathcal{B}(\mu, S)$ are $L$-structures with universes $A(\mu, S)$ and $B(\mu, S), L=\{\leqslant\}$ and $f \leqslant g \Longleftrightarrow f \subset g$. Their cardinality is $2^{<\mu}$.

Because we need to mark the levels, we will temporarily add $\mu$-many unary relation symbols to the vocabulary $\left\{P_{\alpha} \mid \alpha<\mu\right\}$ and interpret them to fix the levels

$$
P_{\alpha}^{\mathcal{A}(\mu, S)}=\{f \in A(\mu, S) \mid \operatorname{dom}(f)=\alpha+1\}
$$

and

$$
P_{\alpha}^{\mathcal{B}(\mu, S)}=\{f \in B(\mu, S) \mid \operatorname{dom}(f)=\alpha+1\} .
$$

In the end we will show how this can be avoided and done with a finite vocabulary. The idea is of the same nature as that of Theorems 5.14, 5.15] and the remark which followed.

If $f: \gamma \rightarrow \mu \times \omega$, denote by $f_{\mu}=\operatorname{pr}_{\mu} \circ f$ and $f_{\omega}=\operatorname{pr}_{\omega} \circ f$. The intention here is that the structures $\mathcal{A}(\mu, S)$ and $\mathcal{B}(\mu, S)$ are trees and that the subtrees $\mathcal{A}_{\alpha}=$ $\left\{f \in \mathcal{A} \mid \operatorname{ran}\left(f_{\mu}\right) \subsetneq \alpha\right\}$ and $\mathcal{B}_{\alpha}=\left\{f \in \mathcal{B} \mid \operatorname{ran}\left(f_{\mu}\right) \subsetneq \alpha\right\}$ are isomorphic if and only if $\alpha \cap S$ contains a cub. If $S$ is complicated enough, we get structures on which $\mathrm{EF}_{\omega_{1}}^{*}$ is not determined.

5.18. Theorem. Let $\mu>\omega_{1}$ and $S \subset S_{\omega}^{\mu}$. If player $\mathbf{I}$ does not have a winning strategy in $G_{\omega}^{\omega_{1}}(S)$ and $S$ contains arbitrarily long $\omega$-cub sets, then he does not have one in $\mathrm{EF}_{\omega_{1}}^{*}(\mathcal{A}(\mu, S), \mathcal{B}(\mu, S))$.

Remark. The existence of arbitrarily long cub sets means that for every $\alpha<\mu$, $\operatorname{cf}(\alpha) \geqslant \omega_{1}$ there exists a subset of $S$ which is $\omega$-closed and of order type $\alpha$.

Proof. For simplicity denote $\mathcal{A}=\mathcal{A}(\mu, S)$ and $\mathcal{B}=\mathcal{B}(\mu, S)$,

$$
\mathcal{A}_{\alpha}=\left\{f \in \mathcal{A} \mid \operatorname{ran}\left(f_{\mu}\right) \subsetneq \alpha\right\},
$$

and similarly

$$
\mathcal{B}_{\alpha}=\left\{f \in \mathcal{B} \mid \operatorname{ran}\left(f_{\mu}\right) \subsetneq \alpha\right\} .
$$

We inform the reader that this proof is probably the longest one in the present article. We will first proof three claims and then complete the actual proof using them.

Claim 0. For each $\beta<\mu$ and $\lambda \in\left\{\omega, \omega_{1}\right\}$, there is an $\alpha \geqslant \beta$ such that $\operatorname{cf}(\alpha)=\lambda$, $\alpha<\mu$, and there is an $\omega$-cub subset of $\alpha \cap S$ of order type $\alpha$. 
Proof of Claim 0 (Sketch). We will give a proof for the case $\lambda=\omega_{1}$, which is longer than the proof for the other case.

W.l.o.g. assume that $\mathrm{cf} \beta=\omega_{1}$ and denote $\alpha_{0}=\beta$. By the assumption, there exists $\alpha_{1} \geqslant \alpha_{0}$ such that there is an $\omega$-closed (but not necessarily unbounded) set of order type $\alpha_{0}$ in $S \cap \alpha_{1}$. If $\alpha_{1}=\alpha_{0}$, then we are done. Otherwise, find $\alpha_{2}>\alpha_{1}$ such that $\alpha_{2} \cap S \backslash \alpha_{1}$ contains an $\omega$-closed set of order type $\alpha_{1}$. Assume we continue doing this until we have an increasing sequence $\left(\alpha_{i}\right)_{i<\omega}$. We could have chosen $\alpha_{i}$ 's so that $\sup _{i} \alpha_{i} \in S$, because if we could not, then this would provide a winning strategy for player $\mathbf{I}$ in $G_{\omega}^{\omega_{1}}$. Let $\alpha_{\omega}=\sup _{i} \alpha_{i}$ and continue until one has $\left(\alpha_{i}\right)_{i<\omega_{1}}$ and $\alpha_{\gamma}=\sup _{i<\gamma} \alpha_{i} \in S$ for all $\gamma<\omega_{1}$ of cofinality $\omega$, which is possible by the same argument as above.

Let $C_{i}$ be the $\omega$-closed subset of $\alpha_{i+1} \backslash \alpha_{i}$ which is of order type $\alpha_{i}$. Denote $\alpha=\sup _{i<\omega_{1}} \alpha_{i}$. We claim that

$$
C=\left\{\alpha_{\gamma} \mid \operatorname{cf} \gamma=\omega\right\} \cup \bigcup_{i<\omega_{1}} C_{i}
$$

is an $\omega$-cub set of order type $\alpha$ in $\alpha \cap S$. Clearly $C \subset S \cap \alpha$, because we chose $\alpha_{i}$ 's such that the limits fall in $S$. Also it is clearly unbounded, since for every $\alpha_{i}$ the set $C_{i}$ is above $\alpha_{i}$. Let $\left(c_{i}\right)_{i<\omega}$ be an increasing sequence of elements of $C$. If there exist $i$ and $m$ so that $c_{j} \in C_{i}$ for all $j>m$, then $\sup _{i} c_{i}$ is in $C_{i}$. Otherwise suppose $c_{i} \in C_{m_{i}}$ and $\left(m_{i}\right)_{i<\omega}$ is increasing. Then $\sup _{i} c_{i}=\alpha_{\sup _{i} m_{i}} \in C$. The order type of $C$ is $\geqslant \sum_{i<\omega_{1}} \alpha_{i} \geqslant \sup _{i} \alpha_{i}=\alpha$, but since $\sup C=\alpha$, we have $\operatorname{OTP}(C)=\alpha$.

A map $g: \alpha \rightarrow \alpha$ is $\omega$-continuous if for every increasing sequence $\left(x_{k}\right)_{k<\omega}$ in $\alpha$ we have $g\left(\bigcup_{k<\omega} x_{k}\right)=\bigcup_{k<\omega} g\left(x_{k}\right)$. Thus the image of such a function is $\omega$-closed. Define $\mathfrak{C}$ to be the set of such functions $h$ :

$$
\mathfrak{C}=\{h: \alpha \rightarrow S \cap \alpha \mid \alpha \in S \text { and } h \text { is } \omega \text {-continuous, increasing and unbounded }\}
$$

and

$$
\mathfrak{C}_{\alpha}=\{h \in \mathfrak{C} \mid \operatorname{dom}(h)<\alpha\} .
$$

Note that by Claim $0 \mathfrak{C}$ is not empty.

Claim 1. For each $h \in \mathfrak{C}$ with $\operatorname{dom}(h)=\alpha$ there exists an isomorphism $F_{h}: \mathcal{A}_{\alpha} \cong$ $\mathcal{B}_{\alpha}$ in such a way that if $h \subset h^{\prime}$, then $F_{h} \subset F_{h^{\prime}}$.

Proof of Claim 1. Let $h: \alpha \rightarrow S \cap \alpha$ be as in the assumption. Then in particular $h$ is an order isomorphism $\alpha \rightarrow h[\alpha]$ and the latter is an $\omega$-closed unbounded subset of $\alpha$. Hence we can write $h^{-1}$ for the inverse $h[\alpha] \rightarrow \alpha$. For defining the isomorphism $F_{h}: \mathcal{A}_{\alpha} \rightarrow \mathcal{B}_{\alpha}$, let $f \in \mathcal{A}_{\alpha}$ be arbitrary, say $f: \delta \rightarrow \alpha \times \omega, \delta<\alpha$. Put

$$
\beta_{f}=\min (\{\beta \mid f(\beta) \notin h[\alpha] \times\{0\}\} \cup\{\delta\}) .
$$

Now for all $\gamma<\beta_{f}$ let $F_{h}(f)(\gamma)=\left(h^{-1}\left(f_{\mu}(\gamma)\right), 0\right)$, and for all $\gamma \geqslant \beta_{f}$ define

$$
F_{h}(f)(\gamma)= \begin{cases}\left(f_{\mu}(\gamma), f_{\omega}(\gamma)+1\right), & \text { if } f_{\mu}\left(\beta_{f}\right) \notin h[\alpha], \\ \left(f_{\mu}(\gamma), f_{\omega}(\gamma)\right)=f(\gamma), & \text { if } f_{\mu}\left(\beta_{f}\right) \in h[\alpha] .\end{cases}
$$

Clearly $F_{h}(f) \in \mathcal{B}_{\alpha}$ and in fact $F_{h}(f): \delta \rightarrow \alpha \times \omega$ (the same domain as that of $f$ ). We will show that $F_{h}$ is an isomorphism. 
(1) $F_{h}$ is one-to-one and onto. It suffices to define a working inverse map: Let $g \in \mathcal{B}_{\alpha}$ be arbitrary, $g: \delta \rightarrow \alpha \times \omega$. Let $\beta_{g}=\min \left(\left\{\beta \mid g_{\omega}(\beta) \neq 0\right\} \cup\{\delta\}\right)$ and let $F^{-1}(g)=u: \delta \rightarrow \alpha \times \omega$ be such that

$$
u(\gamma)= \begin{cases}h(g(\gamma)), & \text { if } \gamma<\beta_{g}, \\ g(\gamma), & \text { if } \gamma \geqslant \beta_{g} \text { and } g_{\mu}\left(\beta_{g}\right) \in h[\alpha], \\ \left(g_{\mu}(\gamma), g_{\omega}(\gamma)-1\right), & \text { if } \gamma \geqslant \beta_{g} \text { and } g_{\mu}\left(\beta_{g}\right) \notin h[\alpha] .\end{cases}
$$

It is not difficult to check that $f \in \mathcal{A}_{\alpha}$ and $F_{h}(u)=g$.

(2) $F_{h}$ preserves ordering and relations $P_{\alpha}$. For the $P_{\alpha}$, it was already mentioned that $\forall f\left[\operatorname{dom}(f)=\operatorname{dom}\left(F_{h}(f)\right)\right]$. Assume $f \leqslant g$. If $\beta_{g} \geqslant \operatorname{dom}(f)$, then for all $\gamma<\operatorname{dom}(f)$ we have $F_{h}(f)(\gamma)=h^{-1}(f(\gamma))=h^{-1}(g(\gamma))=$ $F_{h}(g)(\gamma)$; thus $F_{h}(f) \leqslant F_{h}(g)$. So assume then that $\beta_{g}<\operatorname{dom}(f)$, in which case $\beta_{f}=\beta_{g}$ and $f_{\mu}\left(\beta_{f}\right) \in h[\alpha] \Longleftrightarrow g_{\mu}\left(\beta_{g}\right) \in h[\alpha]$. Hence clearly $F_{h}(f)(\gamma)=F_{h}(g)(\gamma)$ whenever $\beta_{f} \leqslant \gamma<\operatorname{dom}(f)$. The case $\gamma<\beta_{f}$ is as above.

By (1) and (2) $F_{h}$ is an isomorphism.

Assume that $h \subset h^{\prime}$. Then by definition $F_{h^{\prime} \mid \operatorname{dom} h}=F_{h}$, so the claim follows.

$\square$ Claim 1

Claim 2. Let $h \in \mathfrak{C}$ and $\gamma \geqslant \operatorname{dom}(h)$. Then there exists $h^{\prime} \in \mathfrak{C}$ which extends $h$ and $\gamma \leqslant \operatorname{dom}\left(h^{\prime}\right)$.

Proof of Claim 2. Denote $\alpha=\operatorname{dom} h$ and let $\beta$ be an ordinal satisfying

- $\beta>\gamma$,

- $\operatorname{cf}(\beta)=\omega_{1}$,

- there is an $\omega$-cub-set $W \subset S \cap \beta$ of order type $\beta$,

- $h \in \mathfrak{C}_{\beta}$.

This is possible by the assumption of the theorem. Assume $\eta: \beta \rightarrow W$ is an $\omega$ continuous order isomorphism. Let $\alpha_{0}=\min (W \backslash \gamma)$ and

$$
\alpha_{n+1}=\eta\left(\alpha_{n}\right) \text { and } \alpha_{\omega}=\bigcup_{n<\omega} \alpha_{n} .
$$

Then $\eta \uparrow\left(\alpha, \alpha_{\omega}\right)$ is a function from $\left(\alpha, \alpha_{\omega}\right)$ to $W \cap\left(\alpha, \alpha_{\omega}\right)$. Thus we can define

$$
h^{\prime}=h \cup\{(\alpha, \alpha)\} \cup \eta \uparrow\left(\alpha, \alpha_{\omega}\right) .
$$

Then $h^{\prime}: \alpha_{\omega} \rightarrow S \cap \alpha_{\omega}$ (note that because $h \in \mathfrak{C}, \alpha=\operatorname{dom} h \in S$ ) and $h^{\prime} \in$ $\mathfrak{C}_{\beta}$.

For each $\gamma$ let us define a function $K(\gamma): h \mapsto h^{\prime}$, where $h^{\prime}=h$ if $\gamma<\operatorname{dom} h$ and where if $\gamma \geqslant \operatorname{dom} h$, then $h^{\prime}$ is obtained from $h$ using Claim 2 and the choice of $\eta$ (in the proof of Claim 2).

Now let $\tau$ be any strategy for player $\mathbf{I}$ in $\operatorname{EF}_{\omega_{1}}^{\omega_{1}, \omega_{1}}(\mathcal{A}, \mathcal{B})$. For simplicity let us assume without loss of generality that $\tau\left(\left\langle X_{i}\right\rangle_{i<\beta}\right) \subset \tau\left(\left\langle X_{i}\right\rangle_{i<\alpha}\right)$ whenever $\beta<\alpha$.

Recall that $[A \cup B]^{<\mu}=\{F \subset A \cup B|| F \mid<\mu\}$. Define a function $G:[A \cup B]^{<\mu} \rightarrow$ $\mu$ so that $G(F)=\sup \left\{\operatorname{ran}\left(f_{\mu}\right) \mid f \in F\right\}$.

Notation. If $f: X \rightarrow X$ is a function and $J \subset X$, let $f_{\mathrm{cl}}[J]$ denote the closure of $J$ under $f$ :

$$
f_{\mathrm{cl}}[J]=\text { the smallest subset of } X \text { which contains } P \text { and is closed under } f \text {. }
$$


Let $\tau^{*}$ be a strategy of $\mathbf{I}$ in $G_{\omega}^{\omega_{1}}(S)$ which will be defined using $\tau$.

First step:

$$
\tau^{*}(\varnothing)=G(\tau(\varnothing)) .
$$

Next define $\tau^{*}\left(\left\langle y_{i}\right\rangle_{i<\alpha}\right)$ for $\alpha=\beta+1<\omega_{1}$, where $y_{i}$ are answers of $\Pi$ :

If $\beta=0$, then let $h_{0}$ be an arbitrary element of $\mathfrak{C}$, with the property $y_{0}<$ $\operatorname{dom}\left(h_{0}\right)$. Because $y_{0}>\tau^{*}(\varnothing)$ this implies $\tau(\varnothing) \subset \mathcal{A}_{\operatorname{dom}\left(h_{0}\right)} \cup \mathcal{B}_{\operatorname{dom}\left(h_{0}\right)}$. Then (independently of whether $\beta=0$ or not) define

$$
\begin{aligned}
X_{\beta} & =\left(F_{h_{\beta}} \cup F_{h_{\beta}}^{-1}\right)_{\mathrm{cl}}\left[\bigcup_{\delta \leqslant \beta} \tau\left(\left\langle X_{i}\right\rangle_{i<\delta}\right) \cup\left\{\operatorname{id}_{y_{\beta}}\right\}\right], \\
\tau^{*}\left(\left(y_{i}\right)_{i<\alpha}\right) & =G\left(\tau\left(\left\langle X_{i}\right\rangle_{i \leqslant \beta}\right)\right), \\
h_{\alpha} & =K\left(y_{\alpha}\right)\left(h_{\beta}\right) .
\end{aligned}
$$

Finally define $\tau^{*}\left(\left\langle y_{i}\right\rangle_{i<\alpha}\right)$ for $\alpha$ a limit $<\omega_{1}$ :

$$
\begin{aligned}
X_{\alpha} & =\bigcup_{i<\alpha} X_{i} \cup\left\{\operatorname{id}_{y_{\alpha}}\right\}, \\
\tau^{*}\left(\left(y_{i}\right)_{i<\alpha}\right) & =G\left(\tau\left(\left\langle X_{i}\right\rangle_{i<\alpha}\right)\right) \\
h_{\alpha} & =\bigcup_{i<\alpha} h_{i} \text { if } \bigcup_{i<\alpha} \operatorname{dom} h_{i} \in S \text {, i.e. such exists and is otherwise arbitrary. }
\end{aligned}
$$

Now let $\sigma^{*}$ be a strategy of $\boldsymbol{\Pi}$ which beats $\tau^{*}$, and finally the strategy $\sigma$ of $\boldsymbol{\Pi}$ in $\stackrel{*}{\mathrm{EF}_{\omega_{1}}^{\omega_{1}, \omega_{1}}}$ is obtained from $\sigma^{*}$ by induction as follows:

$$
\sigma\left(\left(X_{i}\right)_{i<\alpha}\right)=X_{\alpha} \text { as defined above. }
$$

Because $\sigma^{*}$ beats $\tau^{*}$, it is obvious that $h_{\alpha}$ exists for all limit $\alpha$, since $\bigcup_{i<\alpha} \operatorname{dom} h_{i} \in$ $S$. Thus for all $i<\omega_{1}$ we have $X_{i} \cap \mathcal{A} \cong X_{i} \cap \mathcal{B}$, and moreover the isomorphisms extend each other; i.e.

$$
i<j \Rightarrow X_{i} \subset X_{j} \text { and } F_{i} \subset F_{j},
$$

where $F_{i}$ is the isomorphism between $X_{i} \cap \mathcal{A} \cong X_{i} \cap \mathcal{B}$ and $F_{j}$ is the isomorphism between $X_{j} \cap \mathcal{A} \cong X_{j} \cap \mathcal{B}$. Thus $\sigma$ beats $\tau$, and $\tau$ is not winning.

5.19. Theorem. Let $\mu$ be a cardinal, $S \subset S_{\omega}^{\mu}$ and $\hat{S}=\left\{\alpha \in S_{\omega_{1}}^{\mu} \mid \alpha \cap S\right.$ contains a cub $\}$. If player $\boldsymbol{\Pi}$ does not have a winning strategy in

$$
G_{\omega_{1}}^{\omega_{1}}(\hat{S})
$$

then she does not have one in $\mathrm{EF}_{\omega_{1}}^{*}(\mathcal{A}(\mu, S), \mathcal{B}(\mu, S))$.

Proof. Let $\sigma$ be any strategy of $\Pi$ in $\mathrm{EF}_{\omega_{1}}^{*} \omega_{1}, \omega_{1}(\mathcal{A}(\mu, S), \mathcal{B}(\mu, S))$. Without loss of generality, assume that whenever a sequence $\left(E_{i}\right)_{i<\gamma}$ is played, it holds that $i<j \rightarrow E_{i} \subset E_{j}$.

Let $C$ be the cub set $\{\alpha<\mu \mid \forall \beta<\alpha(\beta+\beta<\alpha)\}$. Let $G:[A \cup B]^{<\mu} \rightarrow \mu$ be as in the proof of the previous theorem and $\hat{G}$ a similar function with a little modification:

$$
\hat{G}(F)=\min \{\alpha \in \hat{S} \cap C \mid \alpha \geqslant G(F))\} .
$$

In the first part it only matters that $\hat{G}(F) \in \hat{S}$ and $\hat{G}(F) \geqslant G(F)$. 
Let $\sigma^{*}$ be the strategy of player $\Pi$ in $G_{\omega_{1}}^{\omega_{1}}(\hat{S})$ which is obtained from $\sigma$ and $\hat{G}$ as follows:

$$
\left.\sigma^{*}\left(\left(\alpha_{i}\right)_{i<\gamma}\right)=\hat{G}\left(\sigma(\underbrace{\left\{\operatorname{id}_{\alpha_{i}+1}\right\}}_{\subset \mathcal{B}})_{i<\gamma}\right)\right) ;
$$

i.e. II imagines that I played the set $\left\{\operatorname{id}_{\alpha_{i}+1}\right\}$ instead of $\alpha_{i}$ in $G_{\omega_{1}}^{\omega_{1}}(\hat{S})$. Let $\tau^{*}$ be the strategy of $\mathbf{I}$ in $G_{\omega_{1}}^{\omega_{1}}(\hat{S})$, which beats $\sigma^{*}$. Then let the strategy $\tau$ be such that if $E_{i} \subset A \cup B$ for each $i<\gamma$ are the moves of $\Pi$ in $\mathrm{EF}_{\omega_{1}}^{\omega_{1}, \omega_{1}}$, then

$$
\left.\tau\left(\left(E_{i}\right)_{i<\gamma}\right)=\left\{\operatorname{id}_{\beta+1}\right\} \subset \mathcal{B}, \text { where } \beta=\tau^{*}\left(\left(\hat{G}\left(E_{i}\right)\right)_{i<\gamma}\right)\right\} .
$$

Assume the players picked $X \subset \mathcal{A} \cup \mathcal{B}$. Because $\tau^{*}$ beats $\sigma^{*}, X \cap \mathcal{B} \subset \mathcal{B}_{G(X)}$ contains an unbounded branch of length $\omega_{1},\left\{\operatorname{id}_{\beta_{i}+1} \mid i<\omega_{1}\right\}$, but there is no unbounded branch of such length in the structure $X \cap \mathcal{A} \subset \mathcal{A}_{G(X)}$ (because there is no $\omega$-cub set in $G(X)$ ).

It remains to show that the unbounded branch $I=\left\{\operatorname{id}_{\beta_{i}+1} \mid i<\omega_{1}\right\}$ would be mapped to an unbounded branch by an isomorphism. For a contradiction assume $F$ to be an isomorphism. It preserves levels, and the level of $\operatorname{id}_{\beta_{i}+1}$ is $\beta_{i}$, i.e. $\operatorname{id}_{\beta_{i}+1} \in P_{\beta_{i}}^{\mathcal{B}}$. So if $F\left(\operatorname{id}_{\beta_{i}+1}\right)=f_{i}$, then $\operatorname{dom}\left(f_{i}\right)=\beta_{i}+1$. Thus

$$
\beta=\sup \{\operatorname{dom}(f) \mid f \in F[I]\}=\bigcup_{i<\omega_{1}} \operatorname{dom}\left(\operatorname{id}_{\beta_{i}+1}\right)=\bigcup_{i<\omega_{1}} \beta_{i}
$$

and its cofinality is $\omega_{1}$. From the definition of $\hat{G}$ it follows that $\beta$ is in $C$. Hence

$$
(\forall \gamma<\beta)(\gamma+\gamma<\beta)
$$

and hence if $\bigcup_{i} \operatorname{pr}_{\mu}\left(\operatorname{ran}\left(f_{i}\right)\right)<\beta$, then we had an increasing function $\beta \rightarrow \alpha$ with $\alpha<\beta$, which is a contradiction.

By the two theorems above it is enough to find a set $S \subset S_{\omega}^{\mu}$ for which

ND1: player I does not have a winning strategy in $G_{\omega}^{\omega_{1}}(S)$,

ND2: $S$ contains arbitrarily long $\omega$-cub sets,

ND3: player $\Pi$ does not have a winning strategy in $G_{\omega_{1}}^{\omega_{1}}(\hat{S})$,

where $\hat{S}=\left\{\alpha \in S_{\omega_{1}}^{\mu} \mid \alpha \cap S\right.$ contains a cub $\}$. Once we have such a set, the game $\mathrm{EF}_{\omega_{1}}^{*}(\mathcal{A}(\mu, S), \mathcal{B}(\mu, S))$ is non-determined.

Stationary sets whose complement satisfies ND1 are called strongly bistationary; see HytSheTuu. A generic set $S \subset S_{\omega}^{\omega_{2}}$ obtained by standard Cohen forcing provides an example of a set which has intended properties ND1 and ND3. A set additionally satisfying ND2 can then be obtained with the use of the following lemma.

5.20. Lemma. Let $S \subset \mu$ satisfy the properties ND1 and ND3. Then there exists $S^{*} \subset \mu$ which satisfies ND1, ND2 and ND3.

Proof. Let $f: \mu \rightarrow \mu$ be the continuous map defined as follows:

$$
f(0)=0, \quad f(\alpha+1)=f(\alpha)+\alpha, \quad f(\gamma)=\bigcup_{\alpha<\gamma} f(\alpha), \text { when } \gamma \text { is a limit. }
$$

This function is clearly continuous. Let

$$
S^{*}=\mu \backslash f[\mu \backslash S] \text {. }
$$

Let us show that $S^{*}$ has the intended properties ND1-ND3. Note that $f[S] \subset S^{*}$. 
ND1: By the assumption, player I does not have a winning strategy in $G_{\omega}^{\omega_{1}}(S)$. Because $f[S] \subset S^{*}$, it is enough to show that $\mathbf{I}$ does not have a winning strategy in $G_{\omega}^{\omega_{1}}(f[S])$. Define $f^{-1}: \mu \rightarrow \mu$ as follows:

$$
f^{-1}(x)=\min (\{y \in \mu \mid f(y) \geqslant x\}) .
$$

Let $\tau$ be any strategy of $\mathbf{I}$ in $G_{\omega}^{\omega_{1}}(f[S])$. Then $\tau^{*}=f^{-1} \circ \tau \circ f$ is a strategy of $\mathbf{I}$ in $G_{\omega}^{\omega_{1}}(S)$. Now by the assumption there is a strategy $\sigma^{*}$ of player $\boldsymbol{\Pi}$ which beats $\tau^{*}$. Now $f \circ \sigma^{*} \circ f^{-1}$ beats $\tau$.

ND2: This is clear from the definitions of $S^{*}$ and $f$.

ND3: For any set $A \subset S_{\omega}^{\mu}$ denote $A^{*}=\mu \backslash f[\mu \backslash A]$ and $\hat{A}=\left\{\alpha \in S_{\omega_{1}}^{\mu} \mid\right.$ $\alpha \cap A$ contains a cub $\}$. Then because $f$ is one-to-one and continuous, we have that

$$
(\hat{S})^{*}=\widehat{\left(S^{*}\right)} \text {. }
$$

Then a similar deduction as for ND1 from the fact that ND3 holds for $S$ follows.

5.21. Theorem. It is consistent that there are structures of cardinality $\aleph_{2}$ such that the game $\mathrm{EF}_{\omega_{1}}^{*}$ is non-determined on these structures.

Proof. Forcing with $\left\{p: \alpha \rightarrow \omega_{2} \mid \alpha<\omega_{2}\right\}$, starting with the ground model in which GCH holds, gives a generic set $S$ such that $\left\{\alpha \in S_{\omega}^{\omega_{2}} \mid \alpha \cap S\right.$ contains cub $\}$ is $\omega_{1}$-bistationary. Note that GCH also holds in the generic extension. Now using GCH it is easy to show the intended properties ND1 and ND3. It is enough to note that the sets $S$ and $\{\alpha \mid S \cap \alpha$ contains cub $\}$ are bistationary. Then by using GCH the players can take closures of each others' strategies and beat them this way. For ND2 one can simply use Lemma 5.20 but in this case it is not necessary.

Conditions ND1 - ND3, i.e. the assumptions of Theorems [5.18 and 5.19, are now satisfied.

Remark. We mentioned in the remark after Theorem 5.9 that we suspect that the linear orders from HytSheVää] would apply to the theorem above if one uses GCH (as we do). But the present method is more general and also provides the following theorems.

5.22. Theorem. Let $\mu=\max \left\{\left(2^{\aleph_{0}}\right)^{+}, \aleph_{4}\right\}$. From ZFC it follows that there are models $\mathcal{A}$ and $\mathcal{B}$ of cardinality $2^{<\mu}=\max \left\{2^{2^{\aleph_{0}}}, 2^{\aleph_{3}}\right\}$ such that $\mathrm{EF}_{\omega_{1}}^{*}(\mathcal{A}, \mathcal{B})$ is nondetermined.

Proof. It was shown in BurMag, Lemma 7.7, that if $\mu>\omega_{3}$ (as is ours), then there is a stationary $X \subset S_{\omega_{2}}^{\mu}$ and sets $D_{\alpha} \subset \alpha$ for each $\alpha \in X$ with the properties

(i) $D_{\alpha}$ is cub in $\alpha$,

(ii) $\operatorname{OTP}\left(D_{\alpha}\right)=\omega_{2}$,

(iii) if $\alpha, \beta \in X$ and $\gamma<\min \{\alpha, \beta\}$ is a limit of both $D_{\alpha}$ and $D_{\beta}$, then $D_{\alpha} \cap \gamma=$ $D_{\beta} \cap \gamma$,

(iv) if $\gamma \in D_{\alpha}$, then $\gamma$ is a limit point of $D_{\alpha}$ if and only if $\gamma$ is a limit ordinal.

Define $X^{\prime}=X \cup\left\{\gamma \mid \exists \alpha>\gamma\left(\gamma \in \lim D_{\alpha}=\right.\right.$ the limit points of $\left.\left.D_{\alpha}\right)\right\}$, and for each $\beta$ in $X^{\prime}$ let

$$
g(\beta)=\min \left\{\gamma \in X \mid \gamma \geqslant \beta \wedge \beta \text { is a limit point of } D_{\gamma}\right\} \in X .
$$

Clearly if $\beta \in X$, then $g(\beta)=\beta$. Then let

$$
C_{\beta}=\beta \cap \lim D_{g(\beta)} \text {. }
$$


We now have the coherence property: If $\beta \in C_{\alpha}$, then $C_{\beta}=\beta \cap C_{\alpha}$. Moreover, each $C_{\alpha}$ is closed, and if $\operatorname{cf}(\alpha) \geqslant \omega_{1}$, then it is unbounded in $\alpha$ and $\operatorname{OTP}\left(C_{\alpha}\right) \leqslant \omega_{2}$. For each $\alpha<\omega_{2}$ define

- $S_{\alpha}=\left\{\beta \in X^{\prime} \mid \operatorname{OTP}\left(C_{\beta}\right)=\alpha\right\}$,

- $S_{\geqslant \alpha}=\bigcup_{\alpha \leqslant \beta<\omega_{2}} S_{\beta}$.

First we observe that for all $\alpha<\omega_{2}, S_{\geqslant \alpha}$ is $\omega$-stationary and $\omega_{1}$-stationary. To see this let $C$ be an $\omega_{1}$-cub set (the $\omega$-case is similar). Because $X$ is stationary, there exists a point $\xi \in X \cap \lim C$. Thus now $C \cap \xi$ is cub in $\xi$. Hence also $C \cap C_{\xi}$ is cub and its order type is obviously $\omega_{2}\left(\xi \in X \subset S_{\omega_{2}}^{\mu}\right.$, and $\operatorname{OTP}\left(C_{\xi}\right)$ is at most $\left.\omega_{2}\right)$. This implies the existence of $\beta \in C_{\xi} \cap C$ such that $C_{\beta}$ is of order type $\geqslant \alpha$ and thus an element of $S_{\geqslant \alpha}$.

Because $S_{\geqslant \alpha}$ is stationary and is a union of $\omega_{2}$ disjoint sets, one of them must be stationary itself. Thus for every $\alpha<\omega_{2}$ there exists $\gamma>\alpha$ such that $S_{\gamma}$ is $\omega$-stationary.

Now we refer to Theorem 3.7 of HytSheTuu which states as applied to our case:

Let $A \subset S_{\omega}^{\mu}$ and assume $A=\bigcup_{i<\omega_{2}} A_{i}$, where each $A_{i}$ is stationary and $A_{i} \cap A_{j}=$ $\varnothing$ if $i \neq j$. Then there is an ordinal $j<\omega_{2}$ such that $\mathbf{I}$ does not have a winning strategy in $G_{\omega}^{\omega_{1}}\left(S_{\omega}^{\mu} \backslash \bigcup_{j \leqslant i<\omega_{2}} A_{i}\right)$.

In our case $A_{i}$ are those sets $\bigcup_{\gamma_{i}<\xi \leqslant \gamma_{i+1}} S_{\omega}^{\mu} \cap S_{\xi}$ where $\left(\gamma_{i}\right)_{i<\omega_{2}}$ is a sequence such that each $S_{\gamma_{i}}$ is $\omega$-stationary. There are $\omega_{2}$ of them as concluded, and all are pairwise disjoint. Let $\gamma$ be such that $\mathbf{I}$ does not have a winning strategy in $G_{\omega}^{\omega_{1}}\left(S_{\omega}^{\mu} \backslash S_{\geqslant \gamma}\right)$ and $S=S_{\omega}^{\mu} \backslash S_{\geqslant \gamma}$. The set $S$ clearly satisfies the intended property ND1.

For ND3 we have to show that player $\boldsymbol{I}$ does not have a winning strategy in $G_{\omega_{1}}^{\omega_{1}}(\hat{S})$, where $\hat{S}=S \cup\left\{\alpha \in S_{\omega_{1}}^{\mu} \mid \alpha \cap S\right.$ contains a cub $\}$. Let us first show that $\left\{\alpha \in S_{\omega_{1}}^{\mu} \mid \alpha \cap S\right.$ does not contain cub $\}$ is $\omega_{1}$-stationary. We know that in the complement of $S$ there is $S_{\geqslant \gamma}$. Let us show that if $C$ is an $\omega_{1}$-cub, then there is such an element $\alpha \in C$ that $S_{\geqslant \gamma} \cap \alpha$ contains a cub, which is more than enough. Let $\beta \in X \cap \lim C$ and let $\alpha$ be the $\left(\gamma+\omega_{1}\right)$ st element of $C_{\beta}$ and $\alpha^{\prime}$ the $\gamma$ th element. Then all points of $C_{\beta} \cap\left[\alpha^{\prime}, \alpha\right)$ are in $S_{\geqslant \gamma}$, because for these points, say $\delta \in C_{\beta} \cap\left[\alpha^{\prime}, \alpha\right)$, we have $C_{\delta}=C_{\beta} \cap \delta$ and it has order type $\geqslant \gamma$. This implies that the set $\left\{\alpha \in S_{\omega_{1}}^{\mu} \mid \alpha \cap S\right.$ does not contain cub $\}$ is stationary.

Assume now that $\sigma$ is a strategy for $\Pi$ in $G_{\omega_{1}}^{\omega_{1}}(\hat{S})$. The set

$$
R=\{\xi \in \mu \mid \xi \text { is closed under } \sigma\}
$$

is an $\omega_{1}$-cub (to see this it is enough to note that $\mu^{<\omega_{1}}=\mu$, which follows from the definition of $\mu$ ). Consequently there is $\alpha \in R \cap\left\{\beta \in S_{\omega_{1}}^{\mu} \mid \beta \cap S\right.$ does not contain cub\}. Player I can now ensure that they play towards $\alpha$, so $\sigma$ cannot be winning. Thus ND1 and ND3 are satisfied, and so by Lemma 5.20 and Theorems 5.18 and 5.19 the game $\mathrm{EF}_{\omega_{1}}^{*}(\mathcal{A}(\mu, S), \mathcal{B}(\mu, S))$ is non-determined.

Remark. At the beginning of this section we promised to show how the vocabulary can be made finite. In order to do this, we have to construct $\mu$ structures $\left(\mathcal{C}_{i}\right)_{i<\mu}$ such that for $i \neq j, \mathbf{I} \uparrow \mathrm{EF}_{\omega_{1}}^{*}\left(\mathcal{C}_{i}, C_{j}\right)$, and add these structures to the levels using one binary relation. This replaces the use of a unary relation $P_{\alpha}$ for each level. During the game player I will make sure that if levels $\alpha$ and $\beta$ are played, then a 'subgame' between $\mathcal{C}_{\alpha}$ in $\mathcal{A}$ and $\mathcal{C}_{\beta}$ in $\mathcal{B}$ is played to show that they are different levels. In the 
end an isomorphism between the picked substructures can only take $\mathcal{C}_{\alpha}$ in $\mathcal{A}$ to $\mathcal{C}_{\alpha}$ in $\mathcal{B}$, as otherwise it contradicts the fact that I won all those 'subgames'.

It remains to find structures $\mathcal{C}_{i}, i<\mu$, for those $\mu$ for which we proved our theorems, i.e. $\mu=\omega_{2}$ and $\mu=\max \left\{\left(2^{\aleph_{0}}\right)^{+}, \aleph_{4}\right\}$.

In the case $\mu=\omega_{2}$ just take all dense linear orders of cardinality $\aleph_{1}$ as in the remark after Theorems 5.14 and 5.15. There are $2^{\aleph_{1}}$, of them and all are different. Because of the small size, then also $\mathbf{I} \uparrow \mathrm{EF}_{\omega_{1}}^{*}\left(\mathcal{C}_{i}, \mathcal{C}_{j}\right)$ if $\mathcal{C}_{i}$ and $\mathcal{C}_{j}$ are two nonisomorphic representatives.

Assume now that $\mu=\max \left\{\left(2^{\aleph_{0}}\right)^{+}, \aleph_{4}\right\}$. It is enough to show that there are $\left(2^{\aleph_{1}}\right)^{++} \geqslant \mu$ models for which the intended property holds.

Let the vocabulary consist of four binary relation symbols and one unary relation $P$ :

$$
L=\left\{R,<,<^{*},<^{\#}, P\right\} .
$$

Let $\mathcal{Q}$ be the disjoint set of well orderings $\left\{\alpha \mid 2^{\omega_{1}} \leqslant \operatorname{OTP}(\alpha)<\left(2^{\omega_{1}}\right)^{+}\right\}$and let $\mathcal{W}$ be the disjoint set of well orderings $\left\{\alpha \mid\left(2^{\omega_{1}}\right)^{+} \leqslant \operatorname{OTP}(\alpha)<\left(2^{\omega_{1}}\right)^{++}\right\}$. Disjoint means that $\alpha \cap \beta=\varnothing$ for all distinct elements $\alpha, \beta \in \mathcal{Q}$ or $\mathcal{W}$. We have:

- $\forall \alpha \in \mathcal{Q}\left(|\alpha|=2^{\aleph_{1}}\right)$,

- $|\mathcal{Q}|=\left(2^{\aleph_{1}}\right)^{+}$,

- $\forall \alpha \in \mathcal{W}\left(|\alpha|=\left(2^{\aleph_{1}}\right)^{+}\right)$,

- $|\mathcal{W}|=\left(2^{\aleph_{1}}\right)^{++}$.

For each $\alpha \in \mathcal{Q}$ let $F_{\alpha}: \mathcal{P}\left(\omega_{1}\right) \rightarrow \alpha$ be a fixed bijection and for each $i \in \mathcal{W}$ let $G_{i}: i \rightarrow \mathcal{Q}$ be another fixed bijection. For each $i \in \mathcal{W}$ define $\mathcal{C}_{i}$ as follows:

- $\operatorname{dom}\left(\mathcal{C}_{i}\right)=\omega_{1} \cup \mathcal{Q}$ (disjoint union),

- $x \mathbb{\#}^{\mathbb{C}_{i}} y \Longleftrightarrow x, y \in \omega_{1} \wedge x<y$ (in $\left.\omega_{1}\right)$,

- $x<^{\mathcal{C}_{i}} y \Longleftrightarrow(\exists \alpha \in \mathcal{Q})(x, y \in \alpha) \wedge x<y$ (in $\left.\alpha\right)$,

- $x<^{* \mathcal{C}_{i}} y \Longleftrightarrow(\exists \alpha, \beta \in \mathcal{Q})\left(G_{i}^{-1}(\alpha)<G_{i}^{-1}(\beta) \wedge x \in \alpha \wedge y \in \beta\right)$,

- $(\alpha, x) \in R^{\mathcal{C}_{i}} \Longleftrightarrow\left(\exists X \in \mathcal{P}\left(\omega_{1}\right)\right)(\exists \beta \in \mathcal{Q})\left(\alpha \in X \wedge x \in \beta \wedge F_{\beta}(X)=x\right)$,

- $P^{\mathcal{C}_{i}}=\omega_{1}$.

Now we claim that $\mathbf{I} \uparrow \mathrm{EF}_{\omega_{1}}^{*} \omega_{1}, \omega_{1}\left(\mathcal{C}_{i}, \mathcal{C}_{j}\right)$ (the game, where the players can choose sets of size $\omega_{1}$; see Theorem 2.11) whenever $i \neq j$. On the first move player $\mathbf{I}$ chooses $P^{\mathcal{C}_{i}} \cup P^{\mathcal{C}_{j}}$. After that player I picks $\alpha$ and $\beta$ in $\mathcal{Q}$ such that $G_{i}^{-1}(\alpha)<G_{i}^{-1}(\beta)$ and $G_{j}^{-1}(\alpha)>G_{j}^{-1}(\beta)$; i.e. $x \in \alpha \wedge y \in \beta \Rightarrow x<^{*} y$ in $\mathcal{C}_{i}$ and $y<^{*} x$ in $\mathcal{C}_{j}$. Such exist, because $i$ and $j$ are non-isomorphic orders. Now player I must make sure that if there is an isomorphism between the played substructures in the end, then it takes $\beta$ in $\mathcal{C}_{i}$ to $\beta$ in $\mathcal{C}_{j}$ and $\alpha$ in $\mathcal{C}_{i}$ to $\alpha$ in $\mathcal{C}_{j}$. This will result in a contradiction, and there cannot be any isomorphism. Because every order $\zeta$ in $\mathcal{Q}$ is different from $\beta$ (provided of course $\zeta \neq \beta$ ), the task is easy for player $\mathbf{I}$. Every time an element is played from an ordering $\zeta$, player I picks two elements $x, y \in \zeta$ and $x^{\prime}, y^{\prime} \in \beta$ such that $x<y, y^{\prime}<x^{\prime}, F_{\zeta}^{-1}(x)=F_{\beta}^{-1}\left(x^{\prime}\right)$ and $F_{\zeta}^{-1}(y)=F_{\beta}^{-1}\left(y^{\prime}\right)$. Because of the relation $R$ it follows that $\beta$ cannot be mapped to $\zeta$ by an isomorphism. Similarly he manages with $\alpha$.

\section{Structures With NON-REFLECTING WinNing STRATEGies}

Let $\mu=\aleph_{\omega \cdot \omega}^{+}$. Put $\mathcal{A}=\mathcal{A}(\mu, S)$ and $\mathcal{B}=\mathcal{B}(\mu, S)$, where $S \subset S_{\omega}^{\mu}$ is the generic set obtained by Cohen forcing starting from a model which satisfies GCH as mentioned 
in the proof of Theorem [5.21, It has the following property: The set

$$
E_{\lambda}=\left\{\alpha \in S_{\lambda}^{\mu} \mid \alpha \cap S \text { contains a cub }\right\}
$$

is $\lambda$-bistationary for each regular $\lambda<\mu$.

For each natural number $n$ let $\alpha_{n}=\aleph_{\omega \cdot n+1}$ (regular) and $\beta_{n}=\aleph_{\omega \cdot(n+1)}$ (singular).

6.1. Theorem (GCH). Let $\lambda<\mu$ be regular (for example $\alpha_{n}$ ). Player $\mathbf{I}$ cannot have a winning strategy in the game $\operatorname{EF}_{\lambda}^{*}(\mathcal{A}, \mathcal{B})$.

Proof. Suppose $\lambda=\alpha_{n}$ for some $n<\omega$. One can show as in Theorem 5.19 that it is enough that player $\Pi$ does not have a winning strategy in $G_{\alpha_{n}}^{\alpha_{n}}\left(E_{\alpha_{n}}\right)$, where $E_{\lambda}$ is defined as in $\varnothing$ for $\lambda=\alpha_{n}$.

Let $\sigma$ be any strategy of $\Pi$ in this game. Then the set

$$
\left\{\alpha \in S_{\alpha_{n}}^{\mu} \mid \alpha \text { is closed under } \sigma\right\}
$$

is an $\alpha_{n}$-cub (by GCH), and thus the complement of $E_{\alpha_{n}}$ intersects it since it is stationary. Player $\mathbf{I}$ can now easily play towards an element in this intersection.

6.2. Theorem (GCH). If $\operatorname{cf}(\lambda)=\omega, \lambda<\mu$ (for example $\lambda=\beta_{n}$ ), then player $\mathbb{\Pi}$ has a winning strategy in the game $\operatorname{EF}_{\lambda}^{*}(\mathcal{A}, \mathcal{B})$.

Proof. Let $\eta: \omega \rightarrow \lambda$ be a cofinal increasing map. As in the proof of Theorem 5.18 there are isomorphisms $F_{\beta}: \mathcal{A}_{\beta} \rightarrow \mathcal{B}_{\beta}$ for each $\beta$ in $E_{\omega_{1}}$. In the game $\mathrm{EF}_{\lambda}^{*}$ player II will play as follows: Assume that $X_{n}$ is the set of already picked elements. By the methods of the proof of Theorem 5.18 she can choose an isomorphism $F_{\beta_{n}}$ such that $\beta_{n}$ is greater than $\sup \left\{\operatorname{dom} f \mid f \in X_{n}\right\}$ and $F_{\beta_{0}} \subset F_{\beta_{1}} \subset \cdots$. Then she chooses the set $\left(F_{\beta_{n}} \cup F_{\beta_{n}}^{-1}\right)\left[X_{n}\right]$. At the end of the game $\bigcup_{k<\omega} F_{\beta_{k}}$ should be a partial isomorphism.

Thus it is consistent that there are models $\mathcal{A}$ and $\mathcal{B}$ and a sequence

$$
\alpha_{0}<\beta_{0}<\alpha_{1}<\beta_{1}<\cdots,
$$

where $\alpha_{n}=\aleph_{\omega \cdot n+1}$ and $\beta_{n}=\aleph_{\omega \cdot(n+1)}$ is such that $\mathcal{A} \mathcal{\psi}_{\alpha_{n}}^{*} \mathcal{B}$ but $\mathcal{A} \sim_{\beta_{n}}^{*} \mathcal{B}$. Note that Example 4.4 gives a similar result in ZFC for ordinals.

\section{REFERENCES}

[BurMag] M. Burke and M. Magidor: Shelah's pcf theory and its applications, Annals of Pure and Applied Logic, 1990.

[Hyt] T. Hyttinen: Games and infinitary languages, Ann. Acad. Sci. Fenn. Series A, I. Math. Dissertationes 64 (1987), 1-32. MR881268 (88a:03086)

[HytSheTuu] T. Hyttinen, S. Shelah and H. Tuuri: Remarks on strong nonstructure theorems, Notre Dame Journal of Formal Logic Volume 34, Number 2, Spring 1993, 157-168. MR.1231281 (94g:03070)

[HytSheVää] T. Hyttinen, S. Shelah and J. Väänänen: More on the Ehrenfeucht-Fraïssé game of length $\omega_{1}$, Fundamenta Mathematicae 175 (2002), no. 1, 79-96. MR 1971240 (2004b:03046)

[Jech] T. Jech: Set Theory, Springer-Verlag, Berlin, Heidelberg, New York, 2003. MR 1940513 (2004g:03071)

[Jen] R. Jensen: The fine structure of the constructible hierarchy, Ann. Math. Logic 4 (1972), 229-308. MR 0309729 (46:8834)

[Kue] D. W. Kueker: Countable approximations and Löwenheim-Skolem theorems, Annals of Math. Logic 11 (1977) 57-103. MR0457191 (56:15406) 
[MekSheVää] A. H. Mekler, S. Shelah and J. Väänänen: The Ehrenfeucht-Fraïssé-game of length $\omega_{1}$, Transactions of the American Mathematical Society, 339 (1993), 567-580. MR.1191613 (94a:03058)

[NadSta] M. Nadel and J. Stavi: $L_{\infty \lambda}$-equivalence, isomorphism and potential isomorphism. Transactions of the American. Math. Soc. 236 (1978), 51-74. MR 0462942 (57:2907)

[She] S. Shelah: Classification Theory, North Holland Publishing Company 1990. MR.1083551 (91k:03085)

Department of Mathematics, University of Helsinki, P.O. Box 68 (Gustav Hällströmin KATU 2B) FI-00014 FINLAND

Department of Mathematics, University of Helsinki, P.O. Box 68 (Gustav Hällströmin KATU 2B) FI-00014 FinLAND 